\title{
BANACH-STONE THEOREMS FOR MAPS PRESERVING COMMON ZEROS
}

\author{
DENNY H. LEUNG AND WEE-KEE TANG
}

\begin{abstract}
Let $X$ and $Y$ be completely regular spaces and $E$ and $F$ be Hausdorff topological vector spaces. We call a linear map $T$ from a subspace of $C(X, E)$ into $C(Y, F)$ a Banach-Stone map if it has the form $T f(y)=S_{y}\left(f(h(y))\right.$ for a family of linear operators $S_{y}: E \rightarrow F$, $y \in Y$, and a function $h: Y \rightarrow X$. In this paper, we consider maps having the property:$$
\cap_{i=1}^{k} Z\left(f_{i}\right) \neq \emptyset \Longleftrightarrow \cap_{i=1}^{k} Z\left(T f_{i}\right) \neq \emptyset
$$

where $Z(f)=\{f=0\}$. We characterize linear bijections with property (Z) between spaces of continuous functions, respectively, spaces of differentiable functions (including $C^{\infty}$ ), as Banach-Stone maps. In particular, we confirm a conjecture of Ercan and Önal:

Suppose that $X$ and $Y$ are realcompact spaces and $E$ and $F$ are Hausdorff topological vector lattices (respectively, $C^{*}$-algebras). Let $T: C(X, E) \rightarrow C(Y, F)$ be a vector lattice isomorphism (respectively, *-algebra isomorphism) such that

$$
Z(f) \neq \emptyset \Longleftrightarrow Z(T f) \neq \emptyset \text {. }
$$

Then $X$ is homeomorphic to $Y$ and $E$ is lattice isomorphic (respectively, $C^{*}$-isomorphic) to $F$.

Some results concerning the continuity of $T$ are also obtained.
\end{abstract}

\section{INTRODUCTION}

Let $X$ and $Y$ be compact Hausdorff spaces. A linear map $T: C(X) \rightarrow$ $C(Y)$ is a surjective isometry if and only if it has the form $T f=w \cdot(f \circ h)$ for some homeomorphism $h: Y \rightarrow X$ and some function $w \in C(Y)$ such that $|w(y)|=1$ for all $y$. This classical result, called the Banach-Stone Theorem, was obtained by Banach [6] for compact metric spaces and extended by Stone [21] to compact Hausdorff spaces. Variations of this result were obtained by Gelfand and Kolmogorov [15] for algebraic isomorphisms and Kaplansky [17] for vector lattice isomorphisms. There has been a great deal of development in this area in the intervening period, including extensions to spaces of vector-valued functions. Let us mention in particular the work on Behrends [7] on $M$-structures and the Banach-Stone property and the theory of separating and biseparating maps (see, e.g., [1, 2, 3, 4, 15, 13, 16, 19]).

Research of the first author was partially supported by AcRF project no. R-146-000086-112. 
We refer to the article [12] for a survey on the many results and research areas arising from the Banach-Stone Theorem.

In a recent article, Ercan and Önal obtained a Banach lattice-valued version of the Banach-Stone Theorem.

Theorem 1. (Ercan and Önal [10]) Let $X$ and $Y$ be compact Hausdorff spaces and $E$ and $F$ be Banach lattices. If there is an onto vector lattice isomorphism $T: C(X, E) \rightarrow C(Y, F)$ such that

$$
Z(f)=\{f=0\} \neq \emptyset \Longleftrightarrow Z(T f) \neq \emptyset,
$$

and $F$ is an $A M$-space with unit, then $X$ is homeomorphic to $Y$ and $E$ and $F$ are isomorphic as Banach lattices.

They further conjectured that the result holds for any Banach lattice $F$. It is easily observed that for a vector lattice isomorphism, condition $\left(\mathrm{Z}_{0}\right)$ is equivalent to

$$
\cap_{i=1}^{k} Z\left(f_{i}\right) \neq \emptyset \Longleftrightarrow \cap_{i=1}^{k} Z\left(T f_{i}\right) \neq \emptyset
$$

for any finite collection of functions $\left(f_{i}\right)_{i=1}^{k}$. Maps satisfying $(\mathrm{Z})$ are termed maps preserving common zeros. In this paper, we undertake a general investigation of linear operators preserving common zeros mapping between certain spaces of vector-valued functions. As a result, we are able to confirm the conjecture of Ercan and Önal in a general setting. We also obtain Banach-Stone type results for mappings between spaces of vector-valued differentiable functions and some theorems on the automatic continuity of such maps. Professor N. C. Wong has informed us that he and his co-authors have independently solved the conjecture of Ercan and Önal 8]. The conjecture was also solved by its proposers Ercan and Önal [11].

In $\S 2$, there is a study of maps preserving common zeros under rather general conditions. Theorem 6 gives a description of such maps, which shows that they are almost of Banach-Stone form. In $\S 3$, we specialize to the case of vector-valued continuous functions, particularly on realcompact spaces. In this case, Theorem 10 provides a complete characterization of maps preserving common zeros. The conjecture of Ercan and Önal [10] follows as a corollary. In $\S 4$, we study maps preserving common zeros mapping between spaces of vector-valued differentiable functions. We are able to characterize such maps as Banach-Stone maps under the general assumption that $E$ and $F$ are Hausdorff topological vector spaces (Theorem 16). Our result also holds for $C^{\infty}$-functions. In the final section, we investigate the continuity properties of the maps considered in $\S 4$. Even without assuming completeness of $E$ and $F$, continuity of the associated map $\Phi$ can be obtained for maps between $C^{m}$-spaces when $m \in \mathbb{N}$. However, this is not longer true if $m=\infty$. To obtain full continuity of the map $T$ requires completeness of $E$ and $F$. These results, summarized in Theorem 23 and Examples 24 and 27. serve to clarify the role played by completeness in theorems regarding automatic continuity. 


\section{MAPS PRESERVING COMMON ZEROS}

If $X$ is a (Hausdorff) completely regular space and $E$ is a Hausdorff topological vector space, let $C(X, E)$ be the set of all continuous functions from $X$ into $E$. It is known that $E$ is completely regular 20 . In particular, every $f \in C(X, E)$ has a unique continuous extension $f^{\beta}: \beta X \rightarrow \beta E$, where $\beta X$ and $\beta E$ are the Stone-Cech compactifications of $X$ and $E$ respectively. The space $C(X, \mathbb{R})$ or $C(X, \mathbb{C})$, as the case may be, is abbreviated to $C(X)$. A vector subspace $A(X)$ of $C(X)$ is said to be almost normal if for every pair of subsets $P$ and $Q$ of $X$ such that $\bar{P}^{\beta X} \cap \bar{Q}^{\beta X}=\emptyset$, there exists $f$ in $A(X)$ such that $f(P) \subseteq\{0\}$ and $f(Q) \subseteq\{1\}$. A vector subspace $A(X, E)$ of $C(X, E)$ is said to be almost normally multiplicative if $A(X, E)$ contains the constant functions and there is an almost normal subspace $A(X)$ of $C(X)$ so that $\varphi \cdot f \in A(X, E)$ whenever $\varphi \in A(X)$ and $f \in A(X, E)$. For any $f \in C(X, E)$, let $Z(f)=\{x \in X: f(x)=0\}$ be the zero set of $f$. If $u \in E$, let $\mathbf{u}$ be the function in $C(X, E)$ with constant value $u$.

In this paper, $X$ and $Y$ will always denote (Hausdorff) completely regular spaces and $E$ and $F$ (nontrivial) Hausdorff topological vector spaces. If $A(X, E)$ and $A(Y, F)$ are subspaces of $C(X, E)$ and $C(Y, F)$ respectively, a linear map $T: A(X, E) \rightarrow A(Y, F)$ is said to preserve common zeros if for any $k \in \mathbb{N}$ and any sequence $\left(f_{i}\right)_{i=1}^{k}$ in $A(X, E)$,

$$
\cap_{i=1}^{k} Z\left(f_{i}\right) \neq \emptyset \Longleftrightarrow \cap_{i=1}^{k} Z\left(T f_{i}\right) \neq \emptyset .
$$

For the rest of the section, $A(X, E)$ and $A(Y, F)$ will denote almost normally multiplicative vector subspaces of $C(X, E)$ and $C(Y, F)$ respectively and $A(X)$ and $A(Y)$ are the corresponding almost normal subspaces of $C(X)$ and $C(Y)$.

Proposition 2. Suppose that $T: A(X, E) \rightarrow A(Y, F)$ is a surjective linear map that preserves common zeros. Then there exist a dense subset $Z$ of $\beta Y$ and a homeomorphism $h: Z \rightarrow X$ so that, for all $y \in Z$ and all $f \in A(X, E)$, there is a net $\left(y_{\alpha}\right)$ in $Y$ converging to $y$ such that

$$
(T f)\left(y_{\alpha}\right)=(T \mathbf{u})\left(y_{\alpha}\right) \text { for all } \alpha \text {, where } u=f(h(y)) \text {. }
$$

In particular, $(T f)^{\beta}(y)=(T \mathbf{u})^{\beta}(y)$.

By $(\mathrm{Z})$ and the compactness of $\beta Y$, the set

$$
Z_{x}=\bigcap_{\substack{f \in A(X, E) \\ f(x)=0}} \overline{Z(T f)}^{\beta Y}
$$

is nonempty for all $x \in X$. Before giving the proof of Proposition 2 , we first establish a number of lemmas.

Lemma 3. If $y \in Z_{x}$, then for all $f \in A(X, E)$, there exists a net $\left(y_{\alpha}\right) \subseteq Y$ converging to $y$ such that

$$
(T f)\left(y_{\alpha}\right)=(T \mathbf{u})\left(y_{\alpha}\right) \text { for all } \alpha,
$$


where $u=f(x)$. As a result,

$$
(T f)^{\beta}(y)=(T \mathbf{u})^{\beta}(y) .
$$

Proof. For any $f \in A(X, E),(f-\mathbf{u})(x)=0$, where $u=f(x)$. Hence $y \in$ $Z_{x} \subseteq \overline{Z(T(f-\mathbf{u}))}^{\beta Y}$. Thus there exists $\left(y_{\alpha}\right) \subseteq Z(T(f-\mathbf{u}))$ converging to $y$. Then

$$
(T f)\left(y_{\alpha}\right)=(T \mathbf{u})\left(y_{\alpha}\right) \text { for all } \alpha \text {. }
$$

Taking limits yield

$$
(T f)^{\beta}(y)=(T \mathbf{u})^{\beta}(y)
$$

Lemma 4. If $x_{1} \neq x_{2}$, then $Z_{x_{1}} \cap Z_{x_{2}}=\emptyset$.

Proof. Suppose that $y_{0} \in Z_{x_{1}} \cap Z_{x_{2}}$. Take any $f \in A(X, E)$ and let $u=$ $f\left(x_{1}\right)$. Since $y_{0} \in Z_{x_{1}}$, by Lemma 3 ,

$$
(T f)^{\beta}\left(y_{0}\right)=(T \mathbf{u})^{\beta}\left(y_{0}\right) \text {. }
$$

There exists $\varphi \in A(X)$ such that $\varphi\left(x_{1}\right)=1$ and $\varphi\left(x_{2}\right)=0$. Since $\varphi f \in$ $A(X, E)$, we may apply Lemma 3 to $\varphi f\left(\right.$ at $\left.x_{1}\right)$ to obtain

$$
(T \mathbf{u})^{\beta}\left(y_{0}\right)=(T(\varphi f))^{\beta}\left(y_{0}\right) .
$$

However, since $y_{0} \in Z_{x_{2}}$, by Lemma 3yet again, $(T(\varphi f))^{\beta}\left(y_{0}\right)=(T \mathbf{v})^{\beta}\left(y_{0}\right)$, where $v=\varphi\left(x_{2}\right) f\left(x_{2}\right)=0$. Thus

$$
(T f)^{\beta}\left(y_{0}\right)=(T \mathbf{v})^{\beta}\left(y_{0}\right)=0 .
$$

This contradicts the surjectivity of $T$, since $\mathbf{w} \in A(Y, F)$ for any $w \in F$ $\{0\}$.

Lemma 5. $\left|Z_{x}\right|=1$ for all $x \in X$.

Proof. Suppose, to the contrary, that there are distinct $y_{0}, y_{1} \in Z_{x}$. Let $U_{0}, U_{1}$ be open neighborhoods of $y_{0}$ and $y_{1}$ in $\beta Y$ respectively whose closures are disjoint. By almost normality of $A(Y)$, there exists $\varphi \in A(Y)$ such that

$$
\varphi=0 \text { on } U_{0} \cap Y \text { and } \varphi=1 \text { on } U_{1} \cap Y \text {. }
$$

Let $v \in F \backslash\{0\}$ and $g=\varphi \cdot \mathbf{v} \in A(Y, F)$. From the fact that $T$ is onto, there exists $f \in A(X, E)$ such that $T f=g$. By Lemma 3 , for $i=0,1$, there exist $\left(y_{\alpha}^{i}\right)$ in $Y$ converging to $y_{i}$ such that

$$
(T \mathbf{u})\left(y_{\alpha}^{i}\right)=(T f)\left(y_{\alpha}^{i}\right)=\varphi\left(y_{\alpha}^{i}\right) v, \text { where } u=f(x) .
$$

Since $\varphi\left(y_{\alpha}^{0}\right)=0$ for sufficiently large $\alpha,(T \mathbf{u})\left(y_{\alpha}^{0}\right)=0$ for such $\alpha$ 's. It follows that $Z(T \mathbf{u}) \neq \emptyset$. By $(Z), Z(\mathbf{u}) \neq \emptyset$ and hence $f(x)=u=0$. On the other hand, $\lim _{\alpha} \varphi\left(y_{\alpha}^{1}\right) v=v \neq 0$. But

$$
\varphi\left(y_{\alpha}^{1}\right) v=(T f)\left(y_{\alpha}^{1}\right)=(T \mathbf{u})\left(y_{\alpha}^{1}\right)=(T \mathbf{0})\left(y_{\alpha}^{1}\right)=0,
$$

a contradiction. 
Proof of Proposition 2. Let $Z=\bigcup_{x} Z_{x}$. Then $Z \subseteq \beta Y$. Define a mapping $h: Z \rightarrow X$ by sending the unique element in $Z_{x}$ to $x$. The mapping $h$ is well-defined by Lemma 4, Clearly $h$ is a bijection and for any $y \in Z$ and $f \in A(X, E)$, Lemma 3 yields a net $\left(y_{\alpha}\right)$ in $Y$ converging to $y$ so that (11) is satisfied. It remains to show that $h$ and $h^{-1}$ are continuous, and that $Z$ is dense in $\beta Y$.

Suppose that $h$ is not continuous. Using the compactness of $\beta X$, there exists $y_{0} \in Z, x^{\prime} \in \beta X$ and a net $\left(y_{\alpha}\right)$ in $Z$ converging to $y_{0}$ such that

$$
h\left(y_{\alpha}\right)=x_{\alpha} \rightarrow x^{\prime} \neq x_{0}=h\left(y_{0}\right) .
$$

Let $U$ be an open neighborhood of $x^{\prime}$ in $\beta X$ such that $\bar{U}^{\beta X}$ does not contain $x_{0}$. Choose $\varphi \in A(X)$ such that $\varphi=0$ in $U \cap X$ and $\varphi\left(x_{0}\right)=1$. For all $f \in A(X, E)$. We have by Lemma 3 that

$$
(T(\varphi f))\left(y_{\alpha}\right)=\left(T \mathbf{u}_{\alpha}\right)\left(y_{\alpha}\right) \text { for all } \alpha,
$$

where $u_{\alpha}=\varphi\left(x_{\alpha}\right) f\left(x_{\alpha}\right)$. Since $u_{\alpha}=\varphi\left(x_{\alpha}\right) f\left(x_{\alpha}\right)=0$ for sufficiently large $\alpha,(T(\varphi f))^{\beta}\left(y_{0}\right)=0$. On the other hand, $(T(\varphi f))^{\beta}\left(y_{0}\right)=(T \mathbf{v})^{\beta}\left(y_{0}\right)$, where $v=\varphi\left(x_{0}\right) f\left(x_{0}\right)=f\left(x_{0}\right)$. Thus $(T f)^{\beta}\left(y_{0}\right)=0$ for all $f \in A(X, E)$, contradicting the surjectivity of $T$.

Suppose that $h^{-1}$ is not continuous. Using the compactness of $\beta Y$, there exists $x_{0} \in X, y^{\prime} \in \beta Y$ and a net $\left(x_{\alpha}\right)$ in $X$ converging to $x_{0}$ such that $h^{-1}\left(x_{\alpha}\right)=y_{\alpha} \rightarrow y^{\prime} \neq y_{0}=h^{-1}\left(x_{0}\right)$. Let $U$ and $V$ be open neighborhoods of $y_{0}$ and $y^{\prime}$ in $\beta Y$ respectively whose closures are disjoint. Choose $\varphi \in A(Y)$ such that $\varphi=1$ in $U \cap Y$ and $\varphi=0$ on $V \cap Y$. Let $v \in F \backslash\{0\}$ and set $g=\varphi \cdot \mathbf{v} \in A(Y, F)$. Since $T$ is surjective, there is an $f \in A(X, E)$ such that $T f=g$. By Lemma 3, for each $\alpha$, there exists a net $\left(y_{\alpha}^{\gamma}\right)_{\gamma}$ in $Y$ converging to $y_{\alpha}$ such that

$$
\begin{aligned}
\left(T \mathbf{u}_{\alpha}\right)\left(y_{\alpha}^{\gamma}\right) & =(T f)\left(y_{\alpha}^{\gamma}\right) \text { for all } \gamma, \text { where } u_{\alpha}=f\left(x_{\alpha}\right), \\
& =g\left(y_{\alpha}^{\gamma}\right)=\varphi\left(y_{\alpha}^{\gamma}\right) v \text { for all } \gamma .
\end{aligned}
$$

For sufficiently large $\alpha$, there exists $\gamma$ where $\varphi\left(y_{\alpha}^{\gamma}\right)=0$. Hence $Z\left(T \mathbf{u}_{\alpha}\right) \neq \emptyset$ for large enough $\alpha$. By $(\mathrm{Z}), Z\left(\mathbf{u}_{\alpha}\right) \neq \emptyset$ and consequently, $f\left(x_{\alpha}\right)=0$. Thus $f\left(x_{0}\right)=0$. Therefore, by Lemma 3 ,

$$
\begin{aligned}
0 & =(T \mathbf{0})^{\beta}\left(y_{0}\right)=(T f)^{\beta}\left(y_{0}\right) \\
& =(\varphi \cdot \mathbf{v})^{\beta}\left(y_{0}\right) \\
& =\varphi^{\beta}\left(y_{0}\right) v \\
& =v \neq 0
\end{aligned}
$$

a contradiction.

Finally, we show that $Z$ is dense in $\beta Y$. If this is not true, then there exists $y_{0} \in \beta Y \backslash \bar{Z}^{\beta Y}$. Let $U$ and $V$ be open neighborhoods of $\bar{Z}^{\beta Y}$ and $y_{0}$ in $\beta Y$ respectively whose closures are disjoint. There exists $\varphi \in A(Y)$ so that $\varphi=0$ on $U \cap Y$ and $\varphi=1$ on $V \cap Y$. Take $v \in F \backslash\{0\}$ and set 
$g=\varphi \cdot \mathbf{v} \in A(Y, F)$. By the surjectivity of $T$, there exists $f \in A(X, E)$ such that $T f=g$. Let $x \in X$ and $y \in Z_{x} \subseteq U$. By Lemma 3 , there exists $\left(y_{\alpha}\right) \subseteq Y$ converging to $y$ such that

$$
\begin{aligned}
(T \mathbf{u})\left(y_{\alpha}\right) & =(T f)\left(y_{\alpha}\right) \text { for all } \alpha, \text { where } u=f(x) \\
& =\varphi\left(y_{\alpha}\right) v .
\end{aligned}
$$

For sufficiently large $\alpha, \varphi\left(y_{\alpha}\right)=0$. Thus $Z(T \mathbf{u}) \neq \emptyset$ and by $(\mathrm{Z}), Z(\mathbf{u}) \neq \emptyset$, which means that $u=0$. Since $x$ is arbitrary, $f=0$. Thus $g=0$, which is absurd.

The next theorem, which is the main result of the section, gives a preliminary description of vector space isomorphisms preserving common zeros. Applications of this theorem in certain cases yield sharp characterizations of said mappings. These applications will be our concern in the subsequent sections.

Theorem 6. Suppose that $T: A(X, E) \rightarrow A(Y, F)$ is a vector space isomorphism that preserves common zeros. Then there are dense subsets $Z$ of $\beta Y$ and $W$ of $\beta X$ and homeomorphisms $h: Z \rightarrow X, k: W \rightarrow Y$ so that $h \cup k^{-1}: Z \cup Y \rightarrow X \cup W$ is a homeomorphism. Moreover, for all $f \in A(X, E), y \in Z$,

$$
(T f)^{\beta}(y)=(T \mathbf{u})^{\beta}(y), u=f(h(y)),
$$

and for all $g \in A(Y, F), x \in W$,

$$
\left(T^{-1} g\right)^{\beta}(x)=\left(T^{-1} \mathbf{v}\right)^{\beta}(x), v=g(k(x)) .
$$

Proof. Applying Proposition 2 to both $T$ and $T^{-1}$, we find dense subsets $Z$ of $\beta Y, W$ of $\beta X$ and homeomorphisms $h: Z \rightarrow X, k: W \rightarrow Y$ such that

$$
(T f)^{\beta}(y)=(T \mathbf{u})^{\beta}(y), u=f(h(y))
$$

whenever $f \in A(X, E), y \in Z$ and

$$
\left(T^{-1} g\right)^{\beta}(x)=\left(T^{-1} \mathbf{v}\right)^{\beta}(x), v=g(k(x))
$$

whenever $g \in A(Y, F), x \in W$.

In the notation of Lemmas 7 and 8, $\tilde{h}_{\mid Z \cup Y}=\tilde{h}_{\mid Z} \cup \tilde{h}_{\mid Y}=h \cup k^{-1}$ is a well-defined map from $Z \cup Y$ to $X \cup W$. By symmetry, $h^{-1} \cup k$ is also a well-defined continuous map. Therefore, $h \cup k^{-1}$ is a homeomorphism. The proof of Theorem 6 is complete.

Lemma 7. There is a continuous extension $\tilde{h}: \beta Y \rightarrow \beta X$ of $h: Z \rightarrow X$.

Proof. If the lemma fails, then there exist $\left(y_{\alpha}^{1}\right),\left(y_{\alpha}^{2}\right)$ in $Z$ converging to $y_{0} \in \beta Y$ such that $h\left(y_{\alpha}^{i}\right) \rightarrow x_{i} \in \beta X, i=1,2$, with $x_{1} \neq x_{2}$. Let $U$ and $V$ be open neighborhoods of $x_{1}$ and $x_{2}$ in $\beta X$ respectively with disjoint closures. There exists $\varphi \in A(X)$ such that $\varphi=0$ on $U \cap X$ and $\varphi=1$ on $V \cap X$. Let $v \in F \backslash\{0\}$ and pick $f \in A(X, E)$ such that $T f=\mathbf{v}$. Applying (2), we find that for all $\alpha$, 
and

$$
v=(T f)^{\beta}\left(y_{\alpha}^{2}\right)=\left(T \mathbf{w}_{\alpha}^{2}\right)^{\beta}\left(y_{\alpha}^{2}\right), \text { where } w_{\alpha}^{2}=f\left(h\left(y_{\alpha}^{2}\right)\right)
$$

$$
(T(\varphi f))^{\beta}\left(y_{\alpha}^{i}\right)=\left(T \mathbf{u}_{\alpha}^{i}\right)^{\beta}\left(y_{\alpha}^{i}\right), \text { where } u_{\alpha}^{i}=(\varphi f)\left(h\left(y_{\alpha}^{i}\right)\right), i=1,2 .
$$

For sufficiently large $\alpha, \varphi\left(h\left(y_{\alpha}^{1}\right)\right)=0$ and $\varphi\left(h\left(y_{\alpha}^{2}\right)\right)=1$ and thus

$$
u_{\alpha}^{i}=\left\{\begin{array}{ccc}
0 & \text { if } & i=1 \\
w_{\alpha}^{2} & \text { if } & i=2 .
\end{array}\right.
$$

It follows that for such $\alpha$,

$$
\begin{aligned}
(T(\varphi f))^{\beta}\left(y_{\alpha}^{i}\right) & =\left\{\begin{array}{cc}
0 & \text { if } i=1, \\
(T f)^{\beta}\left(y_{\alpha}^{2}\right) & \text { if } i=2,
\end{array}\right. \\
& =\left\{\begin{array}{lll}
0 & \text { if } i=1, \\
v & \text { if } i=2 .
\end{array}\right.
\end{aligned}
$$

Upon taking limits, we have $v=(T(\varphi f))^{\beta}\left(y_{0}\right)=0$, a contradiction.

Lemma 8. $\tilde{h}_{\mid Y}=k^{-1}$.

Proof. Suppose that there exists $y_{0} \in Y$ such that $x^{\prime}=\tilde{h}\left(y_{0}\right)$ is different from $x_{0}=k^{-1}\left(y_{0}\right)$. Let $\left(y_{\alpha}\right)$ be a net in $Z$ converging to $y_{0}$ and let $x_{\alpha}=\tilde{h}\left(y_{\alpha}\right)=h\left(y_{\alpha}\right)$. Then $x_{\alpha} \rightarrow x^{\prime}$. Let $U$ and $V$ be respective open neighborhoods of $x^{\prime}$ and $x_{0}$ in $\beta X$ with disjoint closures. Choose $\varphi \in A(X)$ such that $\varphi=0$ on $U \cap X$ and $\varphi=1$ on $V \cap X$ and set $f=\varphi \cdot \mathbf{u}$ for some fixed $u \in E \backslash\{0\}$. For all $\alpha$,

$$
\begin{aligned}
(T f)^{\beta}\left(y_{\alpha}\right) & =\left(T \mathbf{u}_{\alpha}\right)^{\beta}\left(y_{\alpha}\right), \text { where } u_{\alpha}=f\left(x_{\alpha}\right) \\
& =0 \text { when } \alpha \text { is sufficiently large. }
\end{aligned}
$$

Thus $0=(T f)^{\beta}\left(y_{0}\right)=(T f)\left(y_{0}\right)$, since $y_{0} \in Y$. It follows by (3) that

$$
f^{\beta}\left(x_{0}\right)=\left(T^{-1}(T f)\right)^{\beta}\left(x_{0}\right)=\left(T^{-1} \mathbf{0}\right)^{\beta}\left(x_{0}\right)=0,
$$

contradicting the fact that $f(x)=u \neq 0$ for $x \in V \cap X$.

\section{Spaces of CONTINUOUS FunCtions}

In this section, we apply the results of the previous section to the case where $A(X, E)=C(X, E)$ and $A(Y, F)=C(Y, F)$. Obviously, these spaces are almost normally multiplicative. Since the one-point compactification $\mathbb{R}_{\infty}$ of $\mathbb{R}$ is compact, every $f \in C(X)$ has a unique extension to a continuous function $f^{*}: \beta X \rightarrow \mathbb{R}_{\infty}$. Recall that the Hewitt realcompactification $v X$ of $X[14$ is the set

$$
\left\{x \in \beta X: f^{*}(x) \in \mathbb{R} \text { for all } f \in C(X)\right\} .
$$

$X$ is said to be realcompact if $X=v X$.

Proposition 9. Let $A(X, E)=C(X, E)$ and $A(Y, F)=C(Y, F)$ in Proposition 2. Then the set $Z$ obtained in that Proposition is a subset of $v Y$. 
Proof. Suppose, to the contrary, that there exists $y_{0} \in Z \backslash v Y$. Then there exists $\psi \in C(Y)$ such that $\psi(y) \geq 1$ for all $y \in Y$ and $\psi^{*}\left(y_{0}\right)=\infty$. Clearly $\psi^{*}(y) \neq 0$ for all $y \in \beta Y$. Define $\varphi: X \rightarrow \mathbb{R}$ by

$$
\varphi(x)=\frac{1}{\psi^{*}\left(h^{-1}(x)\right)} \quad\left(\text { taking } \frac{1}{\infty}=0\right) .
$$

Then $\varphi \in C(X)$. Let $v \in F \backslash\{0\}$ and choose $f \in C(X, E)$ such that $T f=\psi \cdot \mathbf{v}$.

Claim 1. If $y \in Z$ and $\psi^{*}(y) \in \mathbb{R}$, then $(T(\varphi f))^{\beta}(y)=v$.

Proof of Claim 1. Suppose that $y \in Z$ and $\psi^{*}(y) \in \mathbb{R}$. Let $c=\varphi(h(y))$ and $u=f(h(y))$. By Proposition 2,

$$
\begin{aligned}
(T \mathbf{u})^{\beta}(y) & =(T f)^{\beta}(y) \\
& =(\psi \cdot \mathbf{v})^{\beta}(y) \\
& =\psi^{*}(y) v .
\end{aligned}
$$

Since $c \psi^{*}(y)=\varphi(h(y)) \psi^{*}(y)=1$, we have

$$
\begin{aligned}
v & =c \psi^{*}(y) v \\
& =c(T \mathbf{u})^{\beta}(y) \\
& =(T(c \cdot \mathbf{u}))^{\beta}(y),
\end{aligned}
$$

where the last equality holds because $c \in \mathbb{R}$ and $(T \mathbf{u})^{\beta}(y) \in F$. Finally,

$$
\begin{aligned}
(T(\varphi f))^{\beta}(y) & =(T \mathbf{w})^{\beta}(y), \text { where } w=\varphi(h(y)) f(h(y))=c u, \\
& =(T(c \cdot \mathbf{u}))^{\beta}(y)=v .
\end{aligned}
$$

Claim 2. There exists $\left(y_{\alpha}\right)$ in $Z$ converging to $y_{0}$ such that $\psi^{*}\left(y_{\alpha}\right) \in \mathbb{R}$ for all $\alpha$.

Proof of Claim 2. It is enough to show that for all open neighborhoods $V$ of $y_{0}$ in $\beta Y$, there exists $y \in V \cap Z$ with $\psi^{*}(y) \in \mathbb{R}$. Let $V$ be an open neighborhood of $y_{0}$ in $\beta Y$ and choose $y_{1} \in V \cap Y$. Since $\psi^{*}\left(y_{1}\right)=\psi\left(y_{1}\right) \in \mathbb{R}$, there is an open neighborhood $V_{1}$ of $y_{1}$ in $\beta Y$ such that $\psi^{*}(y) \in \mathbb{R}$ for all $y \in V_{1}$. Now $V \cap V_{1}$ is an open set in $\beta Y$ containing $y_{1}$ and thus is nonempty. Since $Z$ is dense in $\beta Y, Z \cap V \cap V_{1} \neq \emptyset$. If $y \in Z \cap V \cap V_{1}$, then $y \in Z \cap V$ and $\psi^{*}(y) \in \mathbb{R}$.

Returning to the proof of the proposition, let $\left(y_{\alpha}\right)$ be chosen using Claim 2. It follows from Claim 1 that

$$
v=(T(\varphi f))^{\beta}\left(y_{\alpha}\right) \text { for all } \alpha .
$$


Thus, $v=(T(\varphi f))^{\beta}\left(y_{0}\right)$. On the other hand, by Proposition 2 ,

$$
\begin{aligned}
(T(\varphi f))^{\beta}\left(y_{0}\right) & =\left(T \mathbf{u}_{0}\right)^{\beta}\left(y_{0}\right), \text { where } u_{0}=\varphi\left(x_{0}\right) f\left(x_{0}\right) \text { and } x_{0}=h\left(y_{0}\right), \\
& =(T \mathbf{0})^{\beta}\left(y_{0}\right), \text { as } \varphi\left(x_{0}\right)=\frac{1}{\psi^{*}\left(y_{0}\right)}=0 \\
& =0 .
\end{aligned}
$$

Hence $v=0$, contrary to the choice of $v$.

Theorem 10. Let $X$ and $Y$ be realcompact spaces and let $E$ and $F$ be Hausdorff topological vector spaces. Suppose that $T: C(X, E) \rightarrow C(Y, F)$ is a vector space isomorphism that preserves common zeros. Then there are a homeomorphism $h: Y \rightarrow X$ and, for each $y \in Y$, a vector space isomorphism $S_{y}: E \rightarrow F$ so that

$$
T f(y)=S_{y}(f(h(y))) \text { for all } f \in C(X, E) \text { and all } y \in Y \text {. }
$$

Conversely, if a vector space isomorphism $T: C(X, E) \rightarrow C(Y, F)$ has the form (4), then $T$ preserves common zeros.

Proof. By Theorem 6, there exist homeomorphisms $h: Z \rightarrow X, k: W \rightarrow Y$ so that $h \cup k^{-1}: Z \cup Y \rightarrow X \cup W$ is a homeomorphism. According to Proposition 9, $Z \subseteq v Y$. Since $Y$ is realcompact, we deduce that $Z \subseteq Y$ and hence $h$ is a restriction of $k^{-1}$. Similarly, $W \subseteq X$. Then $k^{-1}(Y)=$ $W \subseteq X=h(Z)$. So we must have $X=W$ and $Y=Z$. Therefore, $h$ is a homeomorphism from $Y$ onto $X$.

For each $y \in Y$, define a linear operator $S_{y}: E \rightarrow F$ by

$$
S_{y}(u)=(T \mathbf{u})(y) .
$$

If $u \in \operatorname{ker} S_{y}$, then $y \in Z(T \mathbf{u})$. Thus $Z(T \mathbf{u}) \neq \emptyset$ and hence $Z(\mathbf{u}) \neq \emptyset$. Consequently, $u=0$. The surjectivity of $S_{y}$ follows easily from that of $T$. Therefore, each $S_{y}$ is a linear isomorphism. Since $Z=Y$, it follows from Theorem 6 that

$$
(T f)(y)=S_{y}(f(h(y))) \text { for all } y \in Y .
$$

The converse is clear.

The following corollary of Theorem 10 contains the result conjectured in [10]. The original conjecture was solved by Chen, Chen and Wong [8] and independently by Ercan and Önal [11].

Theorem 11. Let $X$ and $Y$ be realcompact spaces and let $E$ and $F$ be Hausdorff topological vector lattices. Suppose that $T: C(X, E) \rightarrow C(Y, F)$ is a vector lattice isomorphism so that

$$
Z(f) \neq \emptyset \Longleftrightarrow Z(T f) \neq \emptyset \text {. }
$$

Then there is a homeomorphism $h: Y \rightarrow X$ and, for each $y \in Y$, a vector lattice isomorphism $S_{y}: E \rightarrow F$ so that

$$
T f(y)=S_{y}(f(h(y))) \text { for all } f \in C(X, E) \text { and all } y \in Y \text {. }
$$


Proof. We first show that $T$ preserves common zeros. Indeed, if $f_{1}, \cdots, f_{k} \in$ $C(X, E)$, set $f=\left|f_{1}\right| \vee \cdots \vee\left|f_{k}\right|$. It is clear that $Z(f)=\cap_{i=1}^{k} Z\left(f_{i}\right)$. Since $T$ is a lattice isomorphism, $T f=\left|T f_{1}\right| \vee \cdots \vee\left|T f_{k}\right|$. Thus,

$$
\begin{aligned}
\emptyset & \neq \cap_{i=1}^{k} Z\left(f_{i}\right)=Z(f) \\
& \Leftrightarrow Z(T f) \neq \emptyset, \text { by hypothesis, } \\
& \Leftrightarrow \emptyset \neq Z\left(\left|T f_{1}\right| \vee \cdots \vee\left|T f_{k}\right|\right)=\cap_{i=1}^{k} Z\left(T f_{i}\right) .
\end{aligned}
$$

By Theorem 10, we obtain a homeomorphism $h: Y \rightarrow X$ and vector space isomorphisms $S_{y}, y \in Y$, satisfying (4). For any $y \in Y$ and $u \in E$, the equation $(T|\mathbf{u}|)(y)=|T \mathbf{u}|(y)$ shows that $S_{y}(|u|)=\left|S_{y}(u)\right|$. Thus $S_{y}$ is a vector lattice isomorphism.

Theorem 10 holds equally if the scalar field is assumed to be $\mathbb{C}$.

Theorem 12. Let $X$ and $Y$ be realcompact spaces and let $E$ and $F$ be $C^{*}$ algebras. Suppose that $T: C(X, E) \rightarrow C(Y, F)$ is a *-algebra isomorphism so that

$$
Z(f) \neq \emptyset \Longleftrightarrow Z(T f) \neq \emptyset .
$$

Then there is a homeomorphism $h: Y \rightarrow X$ and, for each $y \in Y, a C^{*}$ algebra isomorphism $S_{y}: E \rightarrow F$ so that

$$
T f(y)=S_{y}(f \circ h(y)) \text { for all } f \in C(X, E) \text { and all } y \in Y \text {. }
$$

Proof. If $f_{1}, \cdots, f_{k} \in C(X, E)$, let $f=\sum_{i=1}^{k} f_{i} f_{i}^{*}$. It is clear that $Z(f)=$ $\cap_{i=1}^{k} Z\left(f_{i}\right)$. Since $T$ is a $*$-isomorphism, $T f=\sum_{i=1}^{k} T f_{i}\left(T f_{i}\right)^{*}$. Thus,

$$
\begin{aligned}
\emptyset & \neq \cap_{i=1}^{k} Z\left(f_{i}\right)=Z(f) \\
& \Leftrightarrow Z(T f) \neq \emptyset, \text { by hypothesis, } \\
& \Leftrightarrow \emptyset \neq Z\left(\sum_{i=1}^{k} T f_{i}\left(T f_{i}\right)^{*}\right)=\cap_{i=1}^{k} Z\left(T f_{i}\right) .
\end{aligned}
$$

Therefore $T$ preserves common zeros. The rest of the proof follows along the lines of the proof of Theorem 11 .

\section{Spaces of Differentiable functions}

In this section, we fix $p, q \in \mathbb{N}$ and let $X$ and $Y$ be open subsets of $\mathbb{R}^{p}$ and $\mathbb{R}^{q}$ respectively. The results of $q 2$ are applied to spaces of differentiable functions $C^{m}(X, E)$ and $C^{n}(Y, F)$, where $m, n \in \mathbb{N} \cup\{0, \infty\}$. Here $C^{m}(X, E)$ denotes the space of functions from $X$ into $E$ having continuous partial derivatives of all order $<m+1(\infty+1=\infty)$. Note that the spaces $C^{m}(X)$ and $C^{n}(Y)$ are almost normal and thus $C^{m}(X, E)$ and $C^{n}(Y, F)$ are almost normally multiplicative.

Lemma 13. Let $X$ and $Y$ be open subsets of $\mathbb{R}^{p}$ and $\mathbb{R}^{q}$ respectively, $p, q \in$ $\mathbb{N}$. Suppose that $Z$ is a dense subspace of $\beta Y$ that is homeomorphic to $X$. Then $Z \subseteq Y$. 
Proof. Suppose that $h: Z \rightarrow X$ is a homeomorphism. If $Z \nsubseteq Y$, then there exists $y_{0} \in Z \backslash Y$ such that $h\left(y_{0}\right)=x_{0} \in X$. For each $n$, let $U_{n}$ denote the open set $h^{-1}\left(B\left(x_{0}, \frac{1}{n}\right)\right)$ in $Z$. Then $U_{n}=V_{n} \cap Z$ for some open subset $V_{n}$ of $\beta Y$. Since $Y$ is locally compact, it is open in $\beta Y$. Thus, $V_{n} \cap Y$ is a nonempty open set in $\beta Y$. Therefore, $V_{n} \cap Y \cap Z \neq \emptyset$. For each $n$, pick $y_{n} \in V_{n} \cap Y \cap Z$. Since $h\left(y_{n}\right) \rightarrow x_{0}$ and $h^{-1}$ is continuous, $\left(y_{n}\right)$ converges to $y_{0}$. We may assume without loss of generality that $\left(y_{n}\right)$ is pairwise distinct and has no accumulation point in $Y$. There exists $g \in C(Y)$ such that $0 \leq g \leq 1$, $g\left(y_{2 n-1}\right)=0$ and $g\left(y_{2 n}\right)=1$ for all $n$. Consider the continuous extension $g^{\#}: \beta Y \rightarrow[0,1]$ of $g$. By the continuity of $g^{\#}$,

$$
1=\lim _{n \rightarrow \infty} g\left(y_{2 n}\right)=g^{\#}\left(y_{0}\right)=\lim _{n \rightarrow \infty} g\left(y_{2 n-1}\right)=0,
$$

a contradiction.

Lemma 14. Let $\varphi$ be a real-valued function on $Y$ and $f$ be a function in $C(Y, F)$ that is never zero. Assume that $\lim _{y \rightarrow y_{0}} \varphi(y) f(y)=v$ exists. Then $\lim _{y \rightarrow y_{0}} \varphi(y)=a$ exists and $a \cdot f\left(y_{0}\right)=v$.

Proof. We first show that $\varphi$ is bounded in a neighborhood of $y_{0}$. Suppose otherwise. Then there is a sequence $\left(y_{n}\right)$ converging nontrivially to $y_{0}$ such that $\left|\varphi\left(y_{n}\right)\right| \geq n$ for all $n$. Since $\lim _{y \rightarrow y_{0}} \varphi(y) f(y)=v$ exists, for any circled neighborhood $U$ of $0, \varphi\left(y_{n}\right) f\left(y_{n}\right) \in v+U$ for sufficiently large $n$. Thus

$$
f\left(y_{n}\right)-\frac{v}{\varphi\left(y_{n}\right)} \in \frac{1}{\varphi\left(y_{n}\right)} U \subseteq U
$$

for sufficiently large $n$. Hence $\lim _{n \rightarrow \infty}\left(f\left(y_{n}\right)-\frac{v}{\varphi\left(y_{n}\right)}\right)=0$. By the continuity of $f, f\left(y_{0}\right)=0$, a contradiction.

Since $\varphi$ is bounded in a neighborhood of $y_{0}$, every sequence $\left(y_{n}\right)$ that converges nontrivially to $y_{0}$ has a subsequence $\left(y_{n_{k}}\right)$ such that $\left(\varphi\left(y_{n_{k}}\right)\right)$ converges. Suppose that $\left(y_{n}\right)$ and $\left(z_{n}\right)$ are sequences converging nontrivially to $y_{0}$ such that

$$
\lim _{n \rightarrow \infty} \varphi\left(y_{n}\right)=L_{1} \text { and } \lim _{n \rightarrow \infty} \varphi\left(z_{n}\right)=L_{2} .
$$

Then

$$
L_{1} f\left(y_{0}\right)=\lim _{n \rightarrow \infty} \varphi\left(y_{n}\right) f\left(y_{n}\right)=\lim _{n \rightarrow \infty} \varphi\left(z_{n}\right) f\left(z_{n}\right)=L_{2} f\left(y_{0}\right) .
$$

Since $f\left(y_{0}\right) \neq 0, L_{1}=L_{2}$. Hence $\lim _{y \rightarrow y_{0}} \varphi(y)=a$ exists. Clearly $a \cdot f\left(y_{0}\right)=$ $v$.

Let $r \in \mathbb{N}$. A multi-index $\lambda$ is an $r$-tuple $\left(\lambda_{1}, \ldots, \lambda_{r}\right)$ with entries in $\mathbb{N} \cup\{0\}$, which will also be regarded as a vector in $\mathbb{R}^{r}$. The order of $\lambda$ is $|\lambda|=\lambda_{1}+\cdots+\lambda_{r}$. If $f$ is a function of $r$ variables, we denote by $\partial^{\lambda} f$ the partial derivative $\left(\partial^{1}\right)^{\lambda_{1}} \cdots\left(\partial^{r}\right)^{\lambda_{r}} f$.

Lemma 15. Let $n \in \mathbb{N} \cup\{0\}$. If $\varphi$ is real-valued function on $Y$ and $\varphi f \in$ $C^{n}(Y, F)$ for some $f \in C^{n}(Y, F)$ that is never zero, then $\varphi \in C^{n}(Y)$. 
Proof. The case $n=0$ follows easily from Lemma 14. We prove the remaining cases by induction. Assume that $n=1$. Let $\lambda$ be a multi-index with $|\lambda|=1$. For all $y_{0} \in Y$ and all $t \neq 0$, set $y_{t}=y_{0}+t \lambda$. Then

$$
\begin{aligned}
& \lim _{t \rightarrow 0} \frac{\varphi\left(y_{t}\right)-\varphi\left(y_{0}\right)}{t} f\left(y_{t}\right) \\
& =\lim _{t \rightarrow 0}\left\{\frac{\varphi\left(y_{t}\right) f\left(y_{t}\right)-\varphi\left(y_{0}\right) f\left(y_{0}\right)}{t}-\varphi\left(y_{0}\right) \frac{f\left(y_{t}\right)-f\left(y_{0}\right)}{t}\right\}
\end{aligned}
$$

exists and is equal to

$$
\partial^{\lambda}(\varphi f)\left(y_{0}\right)-\varphi\left(y_{0}\right) \partial^{\lambda} f\left(y_{0}\right)
$$

By Lemma 14, $\partial^{\lambda} \varphi\left(y_{0}\right)=\lim _{t \rightarrow 0} \frac{\varphi\left(y_{t}\right)-\varphi\left(y_{0}\right)}{t}$ exists and

$$
\partial^{\lambda} \varphi\left(y_{0}\right) \cdot f\left(y_{0}\right)=\partial^{\lambda}(\varphi f)\left(y_{0}\right)-\varphi\left(y_{0}\right) \partial^{\lambda} f\left(y_{0}\right) .
$$

From the case $n=0$, we know that $\varphi$ is continuous on $Y$. Together with the assumptions that $\partial^{\lambda}(\varphi f)$ and $\partial^{\lambda} f$ are continuous and that $f$ is never zero, we can deduce using (5) and Lemma 14 that $\partial^{\lambda} \varphi$ is continuous. Since this is true for all multi-indices $\lambda$ with $|\lambda|=1$, we conclude that $\varphi \in C^{1}(X)$.

Suppose that the lemma is true for some integer $n \geq 1$. Assume that $f, \varphi f \in C^{n+1}(Y, F)$, with $f$ never zero on $Y$. By the inductive hypothesis, $\varphi \in C^{n}(Y)$. Also, for any multi-index $\lambda$ with $|\lambda|=1$, we have by (5)

$$
\left(\partial^{\lambda} \varphi\right) \cdot f=\partial^{\lambda}(\varphi f)-\varphi \partial^{\lambda} f .
$$

In particular, $\partial^{\lambda} \varphi \cdot f \in C^{n}(Y, F)$. By the inductive hypothesis, $\partial^{\lambda} \varphi \in$ $C^{n}(Y)$. Hence $\varphi \in C^{n+1}(Y)$.

Theorem 16. Let $X$ and $Y$ be open subsets of $\mathbb{R}^{p}$ and $\mathbb{R}^{q}$ respectively, $p, q \in \mathbb{N}$, and let $E$ and $F$ be Hausdorff topological vector spaces. Suppose that $m, n \in \mathbb{N} \cup\{0, \infty\}$ and $T: C^{m}(X, E) \rightarrow C^{n}(Y, F)$ is a vector space isomorphism so that $T$ preserves common zeros. Then $p=q$ and $m=n$. Moreover, there are a $C^{n}$-diffeomorphism $h: Y \rightarrow X$ and, for each $y \in Y$, a vector space isomorphism $S_{y}: E \rightarrow F$ so that

$$
T f(y)=S_{y}(f \circ h(y)) \text { for all } f \in C^{n}(X, E) \text { and all } y \in Y .
$$

Conversely, if a vector space isomorphism $T: C^{m}(X, E) \rightarrow C^{n}(Y, F)$ has the form (6), then $T$ preserves common zeros.

Proof. Applying Theorem [6 with $A(X, E)=C^{m}(X, E)$ and $A(Y, F)=$ $C^{n}(Y, F)$, there exist homeomorphisms $h: Z \rightarrow X, k: W \rightarrow Y$ so that $h \cup k^{-1}: Z \cup Y \rightarrow X \cup W$ is a homeomorphism. According to Lemma 13, $Z \subseteq Y$ and $W \subseteq X$. Following the arguments as in the proof of Theorem 10, $h: Y \rightarrow X$ is a homeomorphism. Since $X$ and $Y$, which are open subsets of open subsets of $\mathbb{R}^{p}$ and $\mathbb{R}^{q}$ respectively, are homeomorphic, it follows from the Brouwer Domain Invariance Theorem [9, Chapter XVII, Theorem 3.1] that $p=q$. 
We now show that $h \in C^{n}(Y, X)$. Let $v \in E \backslash\{0\}$ be fixed and set $f_{i} \in$ $C^{m}(X, E), i=1, \cdots, p$, to be the function $f_{i}(x)=x_{i} v$ if $x=\left(x_{1}, \cdots, x_{p}\right) \in$ $X$. By Proposition 2, for all $y \in Y$,

$$
\begin{aligned}
\left(T f_{i}\right)(y) & =T \mathbf{u}_{i}(y), \text { where } u_{i}=f_{i}(h(y)) \\
& =\left(T\left(h_{i}(y) \mathbf{v}\right)\right)(y), \text { where } h(y)=\left(h_{1}(y), \cdots, h_{p}(y)\right), \\
& =h_{i}(y) T \mathbf{v}(y)
\end{aligned}
$$

Since $T$ preserves common zeros, $T \mathbf{v}$ is never zero. Applying Lemma 15 to the real-valued functions $h_{i}$ yields that $h_{i} \in C^{n}(Y)$. Hence $h=\left(h_{1}, \cdots, h_{p}\right) \in$ $C^{n}(Y, X)$. Similarly, $h^{-1} \in C^{m}(X, Y)$.

Next, we show that $m=n$. Given $\psi \in C^{m}(Y), \varphi=\psi \circ h^{-1} \in C^{m}(X)$. Fix $u \in E \backslash\{0\}$, and let $f=\varphi \cdot \mathbf{u}$. Then $f \in C^{m}(X, E)$ and hence $T f \in C^{n}(Y, F)$. By Proposition 2 ,

$$
\begin{aligned}
(T f)(y) & =T \mathbf{w}(y), \text { where } w=f(h(y))=\varphi(h(y)) u \\
& =T(\varphi(h(y)) \mathbf{u})(y) \\
& =T(\psi(y) \mathbf{u})(y) \\
& =\psi(y) T \mathbf{u}(y)
\end{aligned}
$$

Since $T \mathbf{u}, T f \in C^{n}(Y, F)$ and $T \mathbf{u}$ is never 0 on $Y$, we conclude from Lemma 15 that $\psi \in C^{n}(Y)$. Hence $C^{m}(Y) \subseteq C^{n}(Y)$ and thus $m \geq n$. By symmetry, $m \leq n$.

For each $y \in Y$, define $S_{y}: E \rightarrow F$ by

$$
S_{y}(u)=(T \mathbf{u})(y) .
$$

From the proof of Theorem[10, we see that $S_{y}$ is a vector space isomorphism that satisfies (6). The converse is clear.

\section{Automatic continuity}

In this section, we investigate the continuity properties of linear isomorphic mappings between spaces of differentiable functions that preserves common zeros. If $T: C^{m}(X, E) \rightarrow C^{n}(Y, F)$ is a linear isomorphism that preserves common zeros, where $X$ and $Y$ are open subsets of $\mathbb{R}^{p}$ and $\mathbb{R}^{q}$ respectively, we have by Theorem 16 that $p=q, m=n$. Also, there are a $C^{m}$-diffeomorphism $h: X \rightarrow Y$ and vector space isomorphisms $S_{y}: E \rightarrow F, y \in Y$, satisfying (66). Define $J: C^{m}(Y, F) \rightarrow C^{m}(X, F)$ by $(J g)(x)=g\left(h^{-1}(x)\right)$. Clearly $J T: C^{m}(X, E) \rightarrow C^{m}(X, F)$ is a vector space isomorphism preserving common zeros and (JTf) $(x)=S_{x}(f(x))$ for all $f \in C^{m}(Y, F)$ and all $x \in X$. Therefore, in considering the continuity of $T$ and the associated map $\Phi: Y \times E \rightarrow F, \Phi(y, u)=S_{y}(u)$, there is no loss of generality in assuming that $X=Y$ and that $h$ is the identity map.

Proposition 17. Let $X$ be an open subset of $\mathbb{R}^{p}, p \in \mathbb{N}, E$ and $F$ be Hausdorff topological vector spaces and $m \in \mathbb{N} \cup\{\infty\}$. Assume that $\Phi$ : $X \times E \rightarrow F$ satisfies 
(1) For all $u \in E, \Phi(\cdot, u)$ belongs to $C^{m}(X, F)$;

(2) For all $x \in X, \Phi(x, \cdot)$ is a linear operator from $E$ into $F$;

(3) $\Phi$ is sequentially continuous.

For all $f \in C^{m}(X, E)$, define $T f(x)=\Phi(x, f(x))$. Then $T f \in C^{m}(X, F)$ and $T$ is a linear operator from $C^{m}(X, E)$ to $C^{m}(X, F)$.

Proof. The proposition holds for $m=\infty$ if it holds for all $m \in \mathbb{N}$. For a fixed $m \in \mathbb{N}$ the proposition is a special case of the following claim:

Claim. If $f \in C^{m}(X, E)$ and $|\lambda| \leq m$, then $\theta_{\lambda}(x)=\Phi\left(x, \partial^{\lambda} f(x)\right)$ belongs to $C^{m-|\lambda|}(X, F)$.

We prove the claim by induction on $m-|\lambda|$. Suppose that $m-|\lambda|=0$. If $f \in C^{m}(X, E)$, then $\partial^{\lambda} f \in C(X, E)$. Thus $\partial^{\lambda} f\left(x_{n}\right) \rightarrow \partial^{\lambda} f\left(x_{0}\right)$ whenever $x_{n} \rightarrow x_{0}$. It follows from the sequential continuity of $\Phi$ that $\theta_{\lambda}\left(x_{n}\right)=$ $\Phi\left(x_{n}, \partial^{\lambda} f\left(x_{n}\right)\right) \rightarrow \Phi\left(x_{0}, \partial^{\lambda} f\left(x_{0}\right)\right)=\theta_{\lambda}\left(x_{0}\right)$. Hence $\theta_{\lambda}$ is sequentially continuous on the metric space $X$. Therefore, $\theta_{\lambda} \in C(X, E)$.

Suppose that the claim is true for $m-|\lambda|=k$. Let $m$ and $\lambda$ be such that $m-|\lambda|=k+1$. If $1 \leq i \leq p$, let $e_{i}$ denote the $i^{\text {th }}$ coordinate unit vector of $\mathbb{R}^{p}$. For $t \neq 0$ and $x_{0} \in X$,

$$
\begin{aligned}
& \frac{\theta_{\lambda}\left(x_{0}+t e_{i}\right)-\theta_{\lambda}\left(x_{0}\right)}{t}=\frac{\Phi\left(x_{0}+t e_{i}, \partial^{\lambda} f\left(x_{0}+t e_{i}\right)\right)-\Phi\left(x_{0}, \partial^{\lambda} f\left(x_{0}\right)\right)}{t} \frac{\Phi\left(x_{0}+t e_{i}, \partial^{\lambda} f\left(x_{0}+t e_{i}\right)\right)-\Phi\left(x_{0}+t e_{i}, \partial^{\lambda} f\left(x_{0}\right)\right)}{t} \\
& \quad+\frac{\Phi\left(x_{0}+t e_{i}, \partial^{\lambda} f\left(x_{0}\right)\right)-\Phi\left(x_{0}, \partial^{\lambda} f\left(x_{0}\right)\right)}{t} \\
&=\Phi\left(x_{0}+t e_{i}, \frac{\partial^{\lambda} f\left(x_{0}+t e_{i}\right)-\partial^{\lambda} f\left(x_{0}\right)}{t}\right)+\frac{g\left(x_{0}+t e_{i}\right)-g\left(x_{0}\right)}{t},
\end{aligned}
$$

where $g(\cdot)=\Phi\left(\cdot, \partial^{\lambda} f\left(x_{0}\right)\right) \in C^{m}(X, F)$, according to (1). If $t_{n} \neq 0, t_{n} \rightarrow 0$, then by $(3)$,

$$
\lim _{n \rightarrow \infty} \frac{\theta_{\lambda}\left(x_{0}+t_{n} e_{i}\right)-\theta_{\lambda}\left(x_{0}\right)}{t_{n}}=\Phi\left(x_{0}, \partial^{i} \partial^{\lambda} f\left(x_{0}\right)\right)+\partial^{i} g\left(x_{0}\right) .
$$

Hence

$$
\partial^{i} \theta_{\lambda}\left(x_{0}\right)=\theta_{\lambda+e_{i}}\left(x_{0}\right)+\partial^{i} g\left(x_{0}\right) .
$$

Since $m-\left|\lambda+e_{i}\right|=m-|\lambda|-1=k, \theta_{\lambda+e_{i}} \in C^{k}(X, F)$ by induction. Also, $\partial^{i} g \in C^{m-1}(X, F) \subseteq C^{k}(X, F)$. It follows that $\partial^{i} \theta_{\lambda} \in C^{k}(X, F)$ for all $1 \leq i \leq p$. Hence $\theta_{\lambda} \in C^{k+1}(X, F)=C^{m-|\lambda|}(X, F)$.

Let $|x|$ denote the Euclidean norm of a vector $x \in \mathbb{R}^{p}$. A $C^{\infty}$-function $\varphi$ : $\mathbb{R}^{p} \rightarrow \mathbb{R}$ is called a $C^{\infty}$-bump if $0 \leq \varphi(x) \leq 1$ for all $x \in \mathbb{R}^{p}, \varphi(x)=1$ if $|x| \leq \frac{1}{2}$ and $\varphi(x)=0$ if $|x| \geq 1$.

Proposition 18. Let $E$ be a Hausdorff topological vector space. Suppose that $\varphi$ is a $C^{\infty}$-bump on $\mathbb{R}^{p},\left(u_{n}\right)$ is a bounded sequence in $E$ and $\left(x_{n}\right)$ is 
a sequence in $\mathbb{R}^{p}$ such that $\left|x_{n+1}-x_{0}\right|<\frac{1}{3}\left|x_{n}-x_{0}\right|<1$ for all $n$. Set $\varphi_{n}(x)=\varphi\left(\frac{x-x_{n}}{r_{n} / 2}\right)$, where $r_{n}=\left|x_{n}-x_{0}\right|$. If $m \in \mathbb{N}$ and $\left(c_{n}\right)$ is a sequence of real numbers such that

$$
\lim _{n \rightarrow \infty} \frac{c_{n}}{r_{n}^{m}}=0,
$$

then $f=\sum c_{n} \varphi_{n} \mathbf{u}_{n} \in C^{m}\left(\mathbb{R}^{p}, E\right)$.

Proof. For a given $n$ and any $a, b$ such that

$$
\frac{3 r_{n+1}}{2}<a<\frac{r_{n}}{2} \text { and } \frac{3 r_{n}}{2}<b<\frac{r_{n-1}}{2}\left(r_{0}=\infty\right),
$$

set $A_{a, b}=\left\{x \in \mathbb{R}^{p}: a<\left|x-x_{0}\right|<b\right\}$. Since for all $k, \varphi_{k}$ is supported on a ball centered at $x_{k}$ with radius $r_{k} / 2$, we see that $f=c_{n} \varphi_{n} \mathbf{u}_{n}$ on $A_{a, b}$. Therefore, $f$ is infinitely differentiable on $A_{a, b}$ and

$$
\partial^{\lambda} f=c_{n} \partial^{\lambda} \varphi_{n} \cdot \mathbf{u}_{n} \text { on } A_{a, b}, \quad|\lambda| \leq m .
$$

It follows easily that $f$ is infinitely differentiable on $\mathbb{R}^{p} \backslash\left\{x_{0}\right\}$ and

$$
\partial^{\lambda} f=\sum_{n} c_{n} \partial^{\lambda} \varphi_{n} \cdot \mathbf{u}_{n} \text { on } \mathbb{R}^{p} \backslash\left\{x_{0}\right\},|\lambda| \leq m .
$$

Claim. If $|\lambda| \leq m$, then $\partial^{\lambda} f\left(x_{0}\right)=0$.

Indeed, if $|\lambda|=0, f\left(x_{0}\right)=\sum c_{n} \varphi_{n}\left(x_{0}\right) u_{n}=0$. Suppose that the claim holds for all $\lambda$ with $|\lambda|=k$ for some fixed $k<m$. Given any $\lambda,|\lambda|=k-1$, $1 \leq i \leq p$ and $t \neq 0$,

$$
\frac{\partial^{\lambda} f\left(x_{0}+t e_{i}\right)-\partial^{\lambda} f\left(x_{0}\right)}{t}=\frac{\partial^{\lambda} f\left(x_{0}+t e_{i}\right)}{t}
$$

by the inductive hypothesis. By (7),

$$
\partial^{\lambda} f\left(x_{0}+t e_{i}\right)=\left\{\begin{array}{cc}
c_{n} \partial^{\lambda} \varphi_{n}\left(x_{0}+t e_{i}\right) u_{n} & \text { if } \\
0 & \frac{r_{n}}{2}<|t|<\frac{3 r_{n}}{2} \\
\text { otherwise. }
\end{array}\right.
$$

Let $U$ be an open neighborhood of zero in $E$. Since $\left(u_{n}\right)$ is a bounded sequence, there exists $\varepsilon>0$ such that $\left(\alpha u_{n}\right) \subseteq U$ for all $|\alpha|<\varepsilon$. Choose $N$ such that $\frac{c_{n}}{\left(r_{n} / 2\right)^{k}}\left\|\partial^{\lambda} \varphi\right\|_{\infty}<\varepsilon$ for all $n>N$. Suppose that $0<|t|<\frac{3 r_{N}}{2}$. Then

$$
\frac{\partial^{\lambda} f\left(x_{0}+t e_{i}\right)}{t}=\left\{\begin{array}{cc}
\frac{c_{n}}{t} \partial^{\lambda} \varphi_{n}\left(x_{0}+t e_{i}\right) u_{n} & \text { if } \\
0 & \frac{r_{n}}{2}<|t|<\frac{3 r_{n}}{2}, n>N \\
\text { otherwise }
\end{array}\right.
$$

But when $\frac{r_{n}}{2}<|t|<\frac{3 r_{n}}{2}$ for some $n>N$,

$$
\begin{aligned}
\left|\frac{c_{n} \partial^{\lambda} \varphi_{n}\left(x_{0}+t e_{i}\right)}{t}\right| & \leq \frac{c_{n}}{r_{n} / 2} \frac{\left\|\partial^{\lambda} \varphi\right\|_{\infty}}{\left(r_{n} / 2\right)^{|\lambda|}} \\
& =\frac{c_{n}}{\left(r_{n} / 2\right)^{k}}\left\|\partial^{\lambda} \varphi\right\|_{\infty}<\varepsilon .
\end{aligned}
$$


Therefore,

$$
\frac{\partial^{\lambda} f\left(x_{0}+t e_{i}\right)-\partial^{\lambda} f\left(x_{0}\right)}{t} \in U \text { if } 0<|t|<\frac{3 r_{N}}{2} .
$$

This shows that $\partial^{i} \partial^{\lambda} f\left(x_{0}\right)=0$. So the claim is verified by induction.

For $|\lambda|=m$ and $x \in \mathbb{R}^{p}$,

$$
\partial^{\lambda} f(x)=\left\{\begin{aligned}
c_{n} \partial^{\lambda} \varphi_{n}(x) u_{n}= & c_{n}\left(\frac{2}{r_{n}}\right)^{|\lambda|} \partial^{\lambda} \varphi\left(\frac{x-x_{n}}{r_{n} / 2}\right) u_{n} \\
& \text { if } \frac{r_{n}}{2}<\left|x-x_{n}\right|<\frac{3 r_{n}}{2}, \\
0 & \text { otherwise. }
\end{aligned}\right.
$$

Since $\left(u_{n}\right)$ is bounded and $\lim _{n \rightarrow \infty} c_{n}\left(\frac{2}{r_{n}}\right)^{|\lambda|}\left\|\partial^{\lambda} \varphi\right\|_{\infty}=0, \lim _{x \rightarrow x_{0}} \partial^{\lambda} f(x)=0$. Hence $\partial^{\lambda} f$ is continuous at $x_{0}$. Thus $f \in C^{m}\left(\mathbb{R}^{p}, E\right)$.

Together with Proposition [17, the next result characterizes when a map $T$, defined in terms of the associated map $\Phi$, sends functions from $C^{m}(X, E)$ to $C^{m}(X, F)$, in the case where $E$ and $F$ are locally convex and $E$ is metrizable.

Theorem 19. Let $X$ be an open subset of $\mathbb{R}^{p}, p \in \mathbb{N}$. Suppose that $E$ and $F$ are Hausdorff topological vector spaces and that $F$ is locally convex. If, for some $m \in \mathbb{N}$, the map $\Phi: X \times E \rightarrow F$ has the property that $T f(x)=$ $\Phi(x, f(x))$ defines a linear operator $T$ from $C^{m}(X, E)$ to $C^{m}(X, F)$. Then $\Phi$ has the following properties.

(1) For all $u \in E, \Phi(\cdot, u)$ belongs to $C^{m}(X, F)$;

(2) For all $x \in X, \Phi(x, \cdot)$ is a linear operator from $E$ into $F$;

$\left(3^{\prime}\right)$ If $\left(x_{n}\right)$ is a sequence in $X$ converging to some $x_{0} \in X$ and $\left(u_{n}\right)$ is a bounded sequence in $E$, then $\left(\Phi\left(x_{n}, u_{n}\right)\right)$ is a bounded sequence in $F$.

Moreover, if $E$ is locally convex metrizable, then $\Phi$ is continuous.

Proof. We first show that the "moreover" statement follows from (1), (2), and $\left(3^{\prime}\right)$. Suppose that $E$ is locally convex metrizable and $\Phi$ is not continuous. Then there exist sequences $\left(x_{n}\right)$ and $\left(u_{n}\right)$ converging to $x_{0}$ and $u_{0}$ in $X$ and $E$ respectively such that $\Phi\left(x_{n}, u_{n}\right) \nrightarrow \Phi\left(x_{0}, u_{0}\right)$. It follows from the local convexity of $F$ that there is a continuous seminorm $\rho$ on $F$ such that

$$
d_{n}=\rho\left(\Phi\left(x_{n}, u_{n}\right), \Phi\left(x_{0}, u_{0}\right)\right) \nrightarrow 0 .
$$

Let $v_{n}=u_{n}-u_{0}$. Then $\left(v_{n}\right)$ converges to 0 . Note that

$$
\begin{aligned}
\Phi\left(x_{n}, v_{n}\right) & -\Phi\left(x_{0}, 0\right)=\Phi\left(x_{n}, u_{n}\right)-\Phi\left(x_{n}, u_{0}\right)-\Phi\left(x_{0}, 0\right) \\
& =\left(\Phi\left(x_{n}, u_{n}\right)-\Phi\left(x_{0}, u_{0}\right)\right)+\left(\Phi\left(x_{0}, u_{0}\right)-\Phi\left(x_{n}, u_{0}\right)\right) .
\end{aligned}
$$

By (1), $\lim _{n \rightarrow \infty}\left(\Phi\left(x_{0}, u_{0}\right)-\Phi\left(x_{n}, u_{0}\right)\right)=0$. Therefore, $\Phi\left(x_{n}, v_{n}\right) \nrightarrow \Phi\left(x_{0}, 0\right)$. This shows that we may assume $u_{0}=0$ without loss of generality. 
By using a subsequence, we may further assume that for some $c>0$, $d_{n}=\rho\left(\Phi\left(x_{n}, u_{n}\right)\right) \geq c$ for all $n$. Since $E$ is locally convex metrizable, there exists a sequence of continuous seminorms $\left(\rho_{k}\right)$ on $E$ which determines the topology on $E$. For all $k, \lim _{n \rightarrow \infty} \rho_{k}\left(u_{n}\right)=0$. Thus there exists a sequence $\left(\alpha_{n}\right)$ of positive numbers diverging to $\infty$ such that $\lim _{n \rightarrow \infty} \alpha_{n} \rho_{k}\left(u_{n}\right)=0$ for all $k$. Now $\left(\alpha_{n} u_{n}\right)$ is a bounded sequence in $E$. But $\rho\left(\Phi\left(x_{n}, \alpha_{n} u_{n}\right)\right)=\alpha_{n} d_{n} \geq$ $\alpha_{n} c \rightarrow \infty$. Hence $\left(3^{\prime}\right)$ fails.

We now turn to proving that $\Phi$ satisfies (1), (2) and $\left(3^{\prime}\right)$. The first two parts are clear. Suppose that $\left(3^{\prime}\right)$ fails. Then there exist sequences $\left(x_{n}\right)$ and $\left(u_{n}\right)$ in $X$ and $E$ respectively, with $\left(x_{n}\right)$ converging to some $x_{0} \in$ $X,\left(u_{n}\right)$ bounded in $E$, such that $\left(\Phi\left(x_{n}, u_{n}\right)\right)$ is unbounded in $F$. Take a continuous seminorm $\rho$ on $F$ such that $\left(\rho\left(\Phi\left(x_{n}, u_{n}\right)\right)\right)$ is unbounded. Using a subsequence if necessary, we may assume that

$$
3 r_{n+1}=3\left|x_{n+1}-x_{0}\right|<\left|x_{n}-x_{0}\right|=r_{n}<1 \text { for all } n
$$

and that $\lim _{n \rightarrow \infty} \rho\left(\Phi\left(x_{n}, u_{n}\right)\right)=\infty$. Set $c_{n}=\frac{1}{\rho\left(\Phi\left(x_{n}, u_{n}\right)\right)} r_{n}^{m}$ for all $n$. By Proposition [18, $f=\sum c_{n} \varphi_{n} \mathbf{u}_{n} \in C^{m}(X, E)$. Therefore, $g(x)=\Phi(x, f(x)) \in$ $C^{m}(X, F)$. By $(2), g(x)=0$ whenever $f(x)=0$. For any $n, a$, and $b$ such that

$$
\frac{3 r_{n+1}}{2}<a<\frac{r_{n}}{2} \text { and } \frac{3 r_{n}}{2}<b<\frac{r_{n-1}}{2},
$$

$f=0$ on $A_{a, b}=\left\{x \in X: a<\left|x-x_{0}\right|<b\right\}$. Thus $g=0$ on $A_{a, b}$. By continuity, we have $\partial^{\lambda} g\left(x_{0}\right)=0$ if $|\lambda| \leq m$. Let $i: F \rightarrow \overline{F_{\rho}}$ be the natural map, where $F_{\rho}$ is the quotient space $F / \rho^{-1}\{0\}$ normed by $\rho$ and $\overline{F_{\rho}}$ is its completion. Then $G=i \circ g \in C^{m}\left(X, \overline{F_{\rho}}\right)$. By Taylor's Formula (see e.g., [18, p.115]), denoting by $\left(x_{n}-x_{0}\right)^{(m)}$ the vector in $\left(\mathbb{R}^{p}\right)^{m}$ with coordinates $x_{n}-x_{0}$ repeated $m$-times,

$$
G\left(x_{n}\right)=G\left(x_{0}\right)+\sum_{k=1}^{m} \frac{D^{(k)} G\left(x_{0}\right)\left(x_{n}-x_{0}\right)^{(m)}}{k !}+E_{n},
$$

where $\lim _{n \rightarrow \infty} \frac{E_{n}}{\left|x_{n}-x_{0}\right|^{m}}=0$. Since $D^{(k)} g\left(x_{0}\right)=0$ for $0 \leq k \leq m$, we have $D^{(k)} G\left(x_{0}\right)=0$ for $0 \leq k \leq m$. Thus $\lim _{n \rightarrow \infty} \frac{G\left(x_{n}\right)}{\left|x_{n}-x_{0}\right|^{m}}=0$. It follows that

$$
\begin{aligned}
0 & =\lim _{n \rightarrow \infty} \frac{\rho\left(g\left(x_{n}\right)\right)}{\left|x_{n}-x_{0}\right|^{m}}=\lim _{n \rightarrow \infty} \frac{\rho\left(\Phi\left(x_{n}, f\left(x_{n}\right)\right)\right)}{r_{n}^{m}} \\
& =\lim _{n \rightarrow \infty} \frac{\rho\left(\Phi\left(x_{n}, c_{n} u_{n}\right)\right)}{r_{n}^{m}}=\lim _{n \rightarrow \infty} \frac{c_{n} \rho\left(\Phi\left(x_{n}, u_{n}\right)\right)}{r_{n}^{m}}=1,
\end{aligned}
$$

a contradiction.

When $E$ and $F$ are locally convex Fréchet spaces, Theorem 19 can be strengthened to yield continuity of the partial derivatives of $\Phi$ with respect to the coordinates of $x$. The results also holds for $m=\infty$. The assumption 
of completeness is crucial here as Examples 24 and 27 will show. The idea for Theorem 20 comes from [3], especially $\S 5$.

Theorem 20. Let $X$ be an open subset of $\mathbb{R}^{p}, p \in \mathbb{N}$, and let $m \in \mathbb{N} \cup\{\infty\}$. Assume that $E$ and $F$ are locally convex Fréchet spaces. Suppose that the map $\Phi: X \times E \rightarrow F$ has the property that $T f(x)=\Phi(x, f(x))$ defines a linear operator $T$ from $C^{m}(X, E)$ into $C^{m}(X, F)$.

(1) If $C^{m}(X, E)$ and $C^{m}(X, F)$ are endowed with complete linear metric topologies that are stronger than the respective topologies of pointwise convergence, then $T$ is continuous;

(2) For any $\lambda$ with $|\lambda|<m+1$, the map $\Phi_{\lambda}: X \times E \rightarrow F$ defined by $\Phi_{\lambda}(x, u)=\left(\partial^{\lambda} T \mathbf{u}\right)(x)$ is continuous. (We adopt the convention $\infty+1=\infty)$.

In the next two lemmas, $E$ and $F$ are locally convex Fréchet spaces.

Lemma 21. Assume that $\Phi$ and $T$ satisfy the hypotheses of Theorem 20 for some $m \in \mathbb{N} \cup\{\infty\}$. Let $\left(u_{n}\right)$ be a bounded sequence in $E$ and $\left(x_{n}\right)$ be a sequence in $\mathbb{R}^{p}$ converging to a point $x_{0}$ such that

$$
3 r_{n+1}<r_{n}<1 \text { for all } n,
$$

where $r_{n}=\left|x_{n}-x_{0}\right|$. If $\left(c_{n}\right)$ is a sequence of real numbers such that

$$
\begin{aligned}
& \lim _{n \rightarrow \infty} \frac{c_{n}}{r_{n}^{m}}=0 \text { if } m \in \mathbb{N}, \\
& \lim _{n \rightarrow \infty} \frac{c_{n}}{r_{n}^{k}}=0 \text { for all } k \in \mathbb{N} \text {, if } m=\infty,
\end{aligned}
$$

then

$$
\begin{aligned}
& \lim _{n \rightarrow \infty} \frac{c_{n} \Phi\left(x_{n}, u_{n}\right)}{r_{n}^{m}}=0 \text { if } m \in \mathbb{N}, \\
& \lim _{n \rightarrow \infty} \frac{c_{n} \Phi\left(x_{n}, u_{n}\right)}{r_{n}^{k}}=0 \text { for all } k \in \mathbb{N} \text {, if } m=\infty .
\end{aligned}
$$

Proof. If $m \in \mathbb{N}$, the conclusion follows since $\left(\Phi\left(x_{n}, u_{n}\right)\right)$ is a bounded sequence by $\left(3^{\prime}\right)$ of Theorem 19. Consider the case $m=\infty$. Let $\varphi$ be a $C^{\infty}$-bump on $\mathbb{R}^{p}$ and set $\varphi_{n}(x)=\varphi\left(\frac{x-x_{n}}{r_{n} / 2}\right)$. According to Proposition 18. $f=\sum c_{n} \varphi_{n} \mathbf{u}_{n} \in C^{\infty}(X, E)$. Let $g(x)=T f(x)=\Phi(x, f(x))$. Then $g \in C^{\infty}(X, F)$. Using the same proof as in Theorem [19, we find that for any continuous seminorm $\rho$ on $F$ and any $k \in \mathbb{N}$,

$$
\begin{aligned}
0 & =\lim _{n \rightarrow \infty} \frac{\rho\left(g\left(x_{n}\right)\right)}{\left|x_{n}-x_{0}\right|^{k}}=\lim _{n \rightarrow \infty} \frac{\rho\left(\Phi\left(x_{n}, f\left(x_{n}\right)\right)\right)}{r_{n}^{k}} \\
& =\lim _{n \rightarrow \infty} \frac{\rho\left(\Phi\left(x_{n}, c_{n} u_{n}\right)\right)}{r_{n}^{k}}=\lim _{n \rightarrow \infty} \rho\left(\frac{c_{n} \Phi\left(x_{n}, u_{n}\right)}{r_{n}^{k}}\right) .
\end{aligned}
$$

The lemma follows since the topology of $F$ is determined by continuous seminorms. 
Lemma 22. Assume that $\Phi$ and $T$ satisfy the hypotheses of Theorem 20 for some $m \in \mathbb{N} \cup\{\infty\}$. Given any compact set $K \subseteq X$, there are only countably many $x \in K$ at which $\Phi(x, \cdot): E \rightarrow F$ is not continuous.

Proof. Let $\rho$ be a continuous seminorm on $F$ and $q: F \rightarrow F_{\rho}$ be the quotient map, where $F_{\rho}$ is the quotient space $F / \rho^{-1}\{0\}$ normed by $\rho$. We claim that $q \circ \Phi(x, \cdot): E \rightarrow F_{\rho}$ is continuous for all but finitely many $x \in K$. Suppose, to the contrary, that there exists a sequence $\left(x_{n}\right)$ converging to $x_{0}$ in $K$ such that $q \circ \Phi\left(x_{n}, \cdot\right)$ is discontinuous for all $n \in \mathbb{N}$. By taking a subsequence if necessary, we may assume that $3 r_{n+1}<r_{n}<1$ for all $n$, where $r_{n}=\left|x_{n}-x_{0}\right|$. Let $c_{n}=r_{n}^{n}$ if $m=\infty$ and $c_{n}=r_{n}^{m}$ if $m \in \mathbb{N}$. Suppose that the topology on $E$ is determined by a sequence of seminorms $\left(\rho_{k}\right)$. For every $n$, by the discontinuity of $q \circ \Phi\left(x_{n}, \cdot\right)$, there exists $u_{n} \in E$ such that $\rho_{k}\left(u_{n}\right) \leq 1,1 \leq k \leq n$, and

$$
\rho\left(\Phi\left(x_{n}, u_{n}\right)\right) \geq \frac{1}{c_{n}}
$$

The sequence $\left(u_{n}\right)$ is bounded in $E$. However,

$$
\lim _{n \rightarrow \infty} \frac{c_{n} \rho\left(\Phi\left(x_{n}, u_{n}\right)\right)}{r_{n}^{k}} \neq 0
$$

for any $k \in \mathbb{N}$, contrary to Lemma 21. This proves the claim. Since the topology of $F$ is determined by countably many seminorms, the lemma follows.

Proof of Theorem 20. (1) It suffices to show that the graph of $T$ is closed. Suppose that $f_{n} \rightarrow f$ in $C^{m}(X, E)$ and $T f_{n} \rightarrow g$ in $C^{m}(X, F)$. Since the topologies on $C^{m}(X, E)$ and $C^{m}(X, F)$ are stronger than the topologies of pointwise convergence, $f_{n}(x) \rightarrow f(x)$ and $T f_{n}(x) \rightarrow g(x)$ for all $x \in X$. At any $x$ where $\Phi(x, \cdot)$ is continuous,

$$
T f_{n}(x)=\Phi\left(x, f_{n}(x)\right) \rightarrow \Phi(x, f(x))=T f(x) .
$$

By Lemma 22, $T f_{n}(x) \rightarrow T f(x)$ for all $x$ in a co-countable subset of $X$. Hence $T f=g$ on a dense subset of $X$. By continuity, $T f=g$.

(2) Endow $C^{m}(X, E)$ and $C^{m}(X, F)$ with the respective topologies of uniform convergence on compact sets of all partial derivatives of order $<$ $m+1$. Then the hypothesis of (1) is satisfied.

If $\left(x_{n}\right)$ and $\left(u_{n}\right)$ are sequences in $X$ and $E$ converging to $x_{0} \in X$ and $u_{0} \in E$ respectively, then the sequence of constant functions $\left(\mathbf{u}_{n}\right)$ converges to $\mathbf{u}_{0}$ in the given topology of $C^{m}(X, E)$. By continuity of $T$, $\left(T \mathbf{u}_{n}\right)$ converges to $T \mathbf{u}_{0}$ in $C^{m}(Y, F)$. Since $K=\left\{x_{n}\right\}_{n=1}^{\infty} \cup\left\{x_{0}\right\}$ is compact, $\partial^{\lambda} T \mathbf{u}_{n} \rightarrow \partial^{\lambda} T \mathbf{u}_{0}$ uniformly on $K$ for all $\lambda,|\lambda|<m+1$. Thus 


$$
\begin{aligned}
& \lim _{n \rightarrow \infty}\left(\left(\partial^{\lambda} T \mathbf{u}_{n}\right)\left(x_{n}\right)-\left(\partial^{\lambda} T \mathbf{u}_{0}\right)\left(x_{n}\right)\right)=0 . \text { Hence } \\
& \begin{aligned}
\Phi_{\lambda}\left(x_{n}, u_{n}\right)-\Phi_{\lambda}\left(x_{0}, u_{0}\right) & =\left(\partial^{\lambda} T \mathbf{u}_{n}\right)\left(x_{n}\right)-\left(\partial^{\lambda} T \mathbf{u}_{0}\right)\left(x_{0}\right) \\
& =\left(\partial^{\lambda} T \mathbf{u}_{n}\right)\left(x_{n}\right)-\left(\partial^{\lambda} T \mathbf{u}_{0}\right)\left(x_{n}\right) \\
& +\left(\partial^{\lambda} T \mathbf{u}_{0}\right)\left(x_{n}\right)-\left(\partial^{\lambda} T \mathbf{u}_{0}\right)\left(x_{0}\right)
\end{aligned}
\end{aligned}
$$

converges to 0 , keeping in mind the continuity of $\partial^{\lambda} T \mathbf{u}_{0}$.

For ease of reference, let us summarize the results from the preceding section and the present one into a unified statement.

Theorem 23. Let $X$ and $Y$ be open subsets of $\mathbb{R}^{p}$ and $\mathbb{R}^{q}$ respectively, $p, q \in \mathbb{N}$, and let $E$ and $F$ be Hausdorff topological vector spaces. Assume that $m, n \in \mathbb{N} \cup\{0, \infty\}$ and $T: C^{m}(X, E) \rightarrow C^{n}(Y, F)$ is a vector space isomorphism so that $T$ preserves common zeros. Then $p=q, m=n$, and there are a $C^{m}$-diffeomorphism $h: Y \rightarrow X$ and a map $\Phi: Y \times E \rightarrow F$ such that

(a) For all $u \in E, \Phi(\cdot, u)$ belongs to $C^{m}(Y, F)$;

(b) For all $y \in Y, \Phi(y, \cdot)$ is a vector space isomorphism from $E$ onto $F$;

(c) $(T f)(y)=\Phi(y, f(h(y)))$ for all $f \in C^{m}(X, E)$ and all $y \in Y$;

(d) If $E$ and $F$ are locally convex metrizable and $m \in \mathbb{N}$, then $\Phi$ is continuous on $Y \times F$. In particular, $E$ and $F$ are isomorphic as topological vector spaces;

(e) If $E$ and $F$ and both locally convex Fréchet, then the conclusion in (d) also holds for $m=\infty$. Furthermore, for any $\lambda$ with $|\lambda|<m+1$, the map $\Phi_{\lambda}: Y \times E \rightarrow F$ defined by $\Phi_{\lambda}(y, u)=\left(\partial^{\lambda} T \mathbf{u}\right)(y)$ is continuous, and $T$ is continuous whenever $C^{m}(X, E)$ and $C^{m}(Y, F)$ are given complete linear metric topologies stronger than the respective topologies of pointwise convergence.

Example 24 demonstrates that neither the continuity of $T$ nor that of $\Phi_{\lambda},|\lambda|>0$, is guaranteed without the completeness of $E$ and $F$. Similarly, Example 27 shows that the the continuity of $\Phi$ itself is not guaranteed if $m=\infty$ and $E$ and $F$ are not assumed to be complete. Denote by $c_{00}$ the space of all finitely supported real sequences endowed with the sup-norm.

Example 24. For any $m \in \mathbb{N}$, there is a map $\Phi: \mathbb{R} \times c_{00} \rightarrow c_{00}$ such that the induced map $T$ given by $T f(x)=\Phi(x, f(x))$ is a linear bijection from $C^{m}\left(\mathbb{R}, c_{00}\right)$ onto itself. However, there is a sequence of functions $\left(f_{n}\right)$ in $C^{m}\left(\mathbb{R}, c_{00}\right)$ so that $\left(f_{n}^{(k)}\right)_{n}$ converges uniformly to 0 for all $k \leq m$, but $\left(\left(T f_{n}\right)^{(k)}(0)\right)_{n}$ does not converge to 0 for any $k, 1 \leq k \leq m$. Furthermore, the maps $\Phi_{k}(0, \cdot), 1 \leq k \leq m$, defined as in part (e) Theorem 23, are not bounded.

If $f \in C^{m}\left(\mathbb{R}, c_{00}\right)$, write $f=\sum f_{i} \mathbf{e}_{i}$, where $\left(e_{i}\right)$ is the unit vector basis of $c_{00}$ and $f_{i} \in C^{m}(\mathbb{R})$. 
Lemma 25. If $f \in C^{m}\left(\mathbb{R}, c_{00}\right)$, then for all $x_{0} \in \mathbb{R}$ and $0 \leq k \leq m$, there exists $i_{0}=i_{0}\left(x_{0}\right) \in \mathbb{N}$ such that

$$
\lim _{x \rightarrow x_{0}} \sup _{i \geq i_{0}} \frac{\left|f_{i}^{(k)}(x)\right|}{\left|x-x_{0}\right|^{m-k}}=0 .
$$

Proof. Let $x_{0} \in \mathbb{R}$ and $0 \leq k \leq m$. Since $f \in C^{m}\left(\mathbb{R}, c_{00}\right)$, for all $0 \leq j \leq m$,

$$
\lim _{x \rightarrow x_{0}} \sup _{i}\left|f_{i}^{(j)}(x)-f_{i}^{(j)}\left(x_{0}\right)\right|=0 .
$$

Since $f^{(j)}\left(x_{0}\right) \in c_{00}$ for all $j \leq m$, there exists $i_{0} \in \mathbb{N}$ such that $f_{i}^{(j)}\left(x_{0}\right)=0$ for all $i \geq i_{0}$ and $0 \leq j \leq m$. For $i \geq i_{0}, x \neq x_{0}$, there exists, by Taylor's Theorem, $\xi=\xi(i, x)$ satisfying $0<\left|\xi-x_{0}\right|<\left|x-x_{0}\right|$, such that

$$
\begin{aligned}
f_{i}^{(k)}(x) & =f_{i}^{(k)}\left(x_{0}\right)+\cdots+\frac{f_{i}^{(m-1)}\left(x_{0}\right)}{(m-1-k) !}\left(x-x_{0}\right)^{m-1-k}+\frac{f_{i}^{(m)}(\xi)}{(m-k) !}\left(x-x_{0}\right)^{m-k} \\
& =\frac{f_{i}^{(m)}(\xi)}{(m-k) !}\left(x-x_{0}\right)^{m-k} .
\end{aligned}
$$

Thus

$$
\frac{\left|f_{i}^{(k)}(x)\right|}{\left|x-x_{0}\right|^{m-k}}=\frac{\left|f_{i}^{(m)}(\xi)\right|}{(m-k) !} .
$$

By (8), for any $\varepsilon>0$, there exists $\delta>0$ such that

$$
\sup _{0<\left|x-x_{0}\right|<\delta} \sup _{i \geq i_{0}}\left|f_{i}^{(m)}(x)\right|=\sup _{0<\left|x-x_{0}\right|<\delta} \sup _{i \geq i_{0}}\left|f_{i}^{(m)}(x)-f_{i}^{(m)}\left(x_{0}\right)\right|<\varepsilon .
$$

Therefore, for $0<\left|x-x_{0}\right|<\delta$,

$$
\sup _{i \geq i_{0}} \frac{\left|f_{i}^{(k)}(x)\right|}{\left|x-x_{0}\right|^{m-k}}<\frac{\varepsilon}{(m-k) !} .
$$

Lemma 26. Let $\left(\varphi_{i}\right)$ be a sequence in $C^{\infty}(\mathbb{R})$. If, for any $x_{0} \in \mathbb{R}$ and $0 \leq j \leq m$, there exists $i_{0} \in \mathbb{N}$ such that

$$
\limsup _{x \rightarrow x_{0}} \sup _{i \geq i_{0}}\left|\varphi_{i}^{(j)}(x)\right|\left|x-x_{0}\right|^{j}=M<\infty
$$

then $\sum f_{i} \varphi_{i} \mathbf{e}_{i} \in C^{m}\left(\mathbb{R}, c_{00}\right)$ whenever $\sum f_{i} \mathbf{e}_{i} \in C^{m}\left(\mathbb{R}, c_{00}\right)$.

Proof. Let $\varepsilon>0$ and $0 \leq j, k \leq m$ with $j+k=m$. According to the hypothesis on $\left(\varphi_{i}\right)$ and Lemma 25, there exists $\delta>0$ and $i_{1} \in \mathbb{N}$ such that

$$
\left|\varphi_{i}^{(j)}(x)\right|\left|x-x_{0}\right|^{j}<M+1 \text { and } \frac{\left|f_{i}^{(k)}(x)\right|}{\left|x-x_{0}\right|^{j}}<\varepsilon
$$

whenever $0<\left|x-x_{0}\right|<\delta$ and $i \geq i_{1}$. Hence

$$
\lim _{x \rightarrow x_{0}} \sup _{i \geq i_{1}}\left|f_{i}^{(k)}(x) \varphi_{i}^{(j)}(x)\right|=0 .
$$


We may also assume that $f_{i}^{(\ell)}\left(x_{0}\right)=0$ for $i \geq i_{1}$ and $0 \leq \ell \leq m$. Thus

$$
\lim _{x \rightarrow x_{0}} \sup _{i \geq i_{1}}\left|f_{i}^{(k)}(x) \varphi_{i}^{(j)}(x)-f_{i}^{(k)}\left(x_{0}\right) \varphi_{i}^{(j)}\left(x_{0}\right)\right|=0 .
$$

Since $f_{i}^{(k)} \varphi_{i}^{(j)}$ is continuous for $1 \leq i<i_{1}$,

$$
\lim _{x \rightarrow x_{0}} \sup _{i}\left|f_{i}^{(k)}(x) \varphi_{i}^{(j)}(x)-f_{i}^{(k)}\left(x_{0}\right) \varphi_{i}^{(j)}\left(x_{0}\right)\right|=0 .
$$

It follows that $\sum\left(f_{i} \varphi_{i}\right)^{(m)} \mathbf{e}_{i} \in C\left(\mathbb{R}, c_{00}\right)$.

Proof of Example 24. Let $\varphi$ be a $C^{\infty}$-bump function. For all $j \in \mathbb{N} \cup\{0\}$, let $C_{j}=\left\|\varphi^{(j)}\right\|_{\infty}$. Set $\varphi_{i}(x)=i \varphi(i x) x^{k}+2$ if $i \in \mathbb{N}_{k}=m \mathbb{N}+k, 1 \leq k \leq m$. Note that

$$
|\varphi(i x)| \leq \chi_{\left[-\frac{1}{i}, \frac{1}{i}\right]}(x) .
$$

In particular $\varphi_{i}(x) \geq 1$ for all $x$. Thus $\psi_{i}(x)=\frac{1}{\varphi_{i}(x)}$ is well-defined for $i \in \mathbb{N}$. We claim that for $x_{0} \in \mathbb{R}$ and $0 \leq j \leq m$, there exists $i_{0} \in \mathbb{N}$ such that

(1) $\limsup _{x \rightarrow x_{0}} \sup _{i \geq i_{0}}\left|\varphi_{i}^{(j)}(x)\right|\left|x-x_{0}\right|^{j}<\infty$

(2) $\limsup _{x \rightarrow x_{0}} \sup _{i \geq i_{0}}\left|\psi_{i}^{(j)}(x)\right|\left|x-x_{0}\right|^{j}<\infty$.

If $x_{0} \neq 0$, there exists $i_{0}$ and a neighborhood $U$ of $x_{0}$ such that $\varphi_{i}$ and $\psi_{i}$ are constant on $U$ for all $i>i_{0}$. Hence (1) and (2) hold. Assume that $x_{0}=0$. It follows from (9) that $1 \leq\left|\varphi_{i}(x)\right| \leq C_{0}|x|^{k-1}+2$ if $i \in \mathbb{N}_{k}$. Therefore (1) and (2) hold for $j=0$. Let $1 \leq j \leq m$ be fixed. If $i \in \mathbb{N}_{k}$, then

$$
\varphi_{i}^{(j)}(x)=i \sum_{\ell=0}^{j \wedge k}\left(\begin{array}{c}
j \\
\ell
\end{array}\right)\left[k(k-1) \cdots(k-\ell+1) x^{k-\ell}\right]\left[i^{j-\ell} \varphi^{(j-\ell)}(i x)\right] .
$$

Thus

$$
\begin{aligned}
\left|\varphi_{i}^{(j)}(x)\right||x|^{j} & \leq \sum_{\ell=0}^{j \wedge k}\left(\begin{array}{c}
j \\
\ell
\end{array}\right) k^{\ell}|x|^{k-1}\left|\varphi^{(j-\ell)}(i x)\right||i x|^{j-\ell+1} \\
& \leq m^{m}|x|^{k-1} \sum_{\ell=0}^{j \wedge k}\left(\begin{array}{c}
j \\
\ell
\end{array}\right) C_{j-\ell} .
\end{aligned}
$$

The last inequality holds because $\varphi^{(j-\ell)}(i x)=0$ when $|i x|>1$. This proves that (1) is satisfied for $1 \leq j \leq m$.

We prove (2) by induction on $j$. Assume that (2) is satisfied for all $j \leq j_{0}$ where $j_{0} \leq m-1$. Note that

$$
0=\left(\varphi_{i} \psi_{i}\right)^{\left(j_{0}+1\right)}=\sum_{\ell=0}^{j_{0}+1}\left(\begin{array}{c}
j_{0}+1 \\
\ell
\end{array}\right) \varphi_{i}^{(\ell)} \psi_{i}^{\left(j_{0}+1-\ell\right)}
$$


Therefore,

$$
\psi_{i}^{\left(j_{0}+1\right)}(x)=\frac{-1}{\varphi_{i}(x)} \sum_{\ell=1}^{j_{0}+1}\left(\begin{array}{c}
j_{0}+1 \\
\ell
\end{array}\right) \varphi_{i}^{(\ell)}(x) \psi_{i}^{\left(j_{0}+1-\ell\right)}(x) .
$$

Since $\left|\frac{1}{\varphi_{i}(x)}\right| \leq 1$,

$$
\left|\psi_{i}^{\left(j_{0}+1\right)}(x)\right||x|^{j_{0}+1} \leq \sum_{\ell=1}^{j_{0}+1}\left(\begin{array}{c}
j_{0}+1 \\
\ell
\end{array}\right)\left|\varphi _ { i } ^ { ( \ell ) } ( x ) \left\|\left.x\right|^{\ell}\left|\psi_{i}^{\left(j_{0}+1-\ell\right)}(x) \| x\right|^{j_{0}+1-\ell} .\right.\right.
$$

The desired inequality (2), with $j_{0}+1$ in place of $j$, follows from (1) and the inductive hypothesis.

By (1), (2) and Lemma 26, the map $T: C^{m}\left(\mathbb{R}, c_{00}\right) \rightarrow C^{m}\left(\mathbb{R}, c_{00}\right)$, $\sum f_{i} \mathbf{e}_{i} \mapsto \sum \varphi_{i} f_{i} \mathbf{e}_{i}$ is a linear bijection. Finally, the sequence $\left(f_{n}\right)=\left(\frac{1}{n} \mathbf{e}_{n}\right)$ in $C^{m}\left(\mathbb{R}, c_{00}\right)$ and the sequences of derivatives $\left(f_{n}^{(k)}\right)_{n}, k \leq m$, converge to 0 uniformly. However, for $1 \leq k \leq m,\left(T f_{n}\right)^{(k)}(0)=k ! e_{n}$ whenever $n \in \mathbb{N}_{k}$. Hence $\lim _{n \rightarrow \infty} \Phi_{k}\left(0, \frac{e_{n}}{n}\right)=\lim _{n \rightarrow \infty} T f_{n}^{(k)}(0) \neq 0$. In particular, $\Phi_{k}(0, \cdot)$ is not bounded.

Example 27. There is a map $\Phi: \mathbb{R} \times c_{00} \rightarrow c_{00}$ such that the induced map $T$ given by $T f(x)=\Phi(x, f(x))$ is a linear bijection from $C^{\infty}\left(\mathbb{R}, c_{00}\right)$ onto itself. However, $\Phi_{k}(0, \cdot)$ is not bounded for any $k \in \mathbb{N} \cup\{0\}$ and there is a sequence of functions $\left(f_{n}\right)$ in $C^{\infty}\left(\mathbb{R}, c_{00}\right)$ so that $\left(f_{n}^{(k)}\right)_{n}$ converges uniformly to 0 for all $k \in \mathbb{N} \cup\{0\}$, but $\left(\left(T f_{n}\right)^{(k)}(0)\right)$ does not converge to 0 for any $k \in \mathbb{N} \cup\{0\}$.

Lemma 28. Let $\left(\varphi_{i}\right)$ be a sequence in $C^{\infty}(\mathbb{R})$. If for any $x_{0} \in \mathbb{R}$ and $k \in \mathbb{N} \cup\{0\}$, there exists $i_{0}=i_{0}(k) \in \mathbb{N}$ such that

$$
\limsup _{x \rightarrow x_{0}} \sup _{i \geq i_{0}}\left|\varphi_{i}^{(k)}(x)\right|\left|x-x_{0}\right|^{k+1}<\infty,
$$

then $\sum f_{i} \varphi_{i} \mathbf{e}_{i} \in C^{\infty}\left(\mathbb{R}, c_{00}\right)$ whenever $\sum f_{i} \mathbf{e}_{i} \in C^{\infty}\left(\mathbb{R}, c_{00}\right)$.

Proof. Given $x_{0} \in \mathbb{R}, k, j \in \mathbb{N} \cup\{0\}$, it follows from Lemma 25 that there exists $i_{1}=i_{1}\left(x_{0}, k, j\right)>i_{0}(j)$ such that $f_{i}^{(k)}\left(x_{0}\right)=0$ if $i \geq i_{0}$ and

$$
\lim _{x \rightarrow x_{0}} \sup _{i \geq i_{1}} \frac{\left|f_{i}^{(k)}(x)\right|}{\left|x-x_{0}\right|^{j+1}}=0 .
$$

It follows readily that $\lim _{x \rightarrow x_{0}} \sup _{i \geq i_{1}}\left|f_{i}^{(k)}(x) \varphi_{i}^{(j)}(x)\right|=0$. The remainder of the argument proceeds as in the proof of Lemma 26

Proof of Example 27. Let $\varphi$ be a $C^{\infty}$-bump function. For all $j \in \mathbb{N} \cup\{0\}$, let $C_{j}=\left\|\varphi^{(j)}\right\|_{\infty}$. Let $\left(\mathbb{N}_{k}\right)_{k \in \mathbb{N}}$ be a partition of $\mathbb{N}$ into infinite sets such that $i \geq k$ if $i \in \mathbb{N}_{k}$. Set $\varphi_{i}(x)=i \varphi(i x) x^{k-1}+2$, if $i \in \mathbb{N}_{k}$ and $\psi_{i}(x)=\frac{1}{\varphi_{i}(x)}$. By arguments similar to those used in the proof of Example 24, one can show that for all $x_{0} \in \mathbb{R}$ and $j \in \mathbb{N} \cup\{0\}$, there exists $i_{0} \in \mathbb{N}$ such that 
(1) $\lim _{x \rightarrow x_{0}} \sup _{i \geq i_{0}}\left|\varphi_{i}^{(j)}(x)\right|\left|x-x_{0}\right|^{j+1}<\infty$,

(2) $\lim _{x \rightarrow x_{0}} \sup _{i \geq i_{0}}\left|\psi_{i}^{(j)}(x)\right|\left|x-x_{0}\right|^{2 j+1}<\infty$.

It follows by Lemma 28 that the map $T: C^{\infty}\left(\mathbb{R}, c_{00}\right) \rightarrow C^{\infty}\left(\mathbb{R}, c_{00}\right)$, $\sum f_{i} \mathbf{e}_{i} \mapsto \sum \varphi_{i} f_{i} \mathbf{e}_{i}$, is a linear bijection. For each $n$, let $f_{n}=\frac{\mathbf{e}_{n}}{n} \in$ $C^{\infty}\left(\mathbb{R}, c_{00}\right)$. Then $\left(f_{n}^{(k)}\right)$ converges uniformly to zero for all $k \in \mathbb{N} \cup\{0\}$. However, $T f_{n}(x)=\frac{\varphi_{n}(x) \mathbf{e}_{n}}{n}=\varphi(n x) x^{k-1}+\frac{2}{n}$ for all $n \in \mathbb{N}_{k} \cdot|x|<1$. Recall that $\varphi_{n}(x)=1$ if $|x| \leq \frac{1}{2 n}$. Therefore, for all $k \in \mathbb{N} \cup\{0\}$ and $n \in \mathbb{N}_{k+1}$

$$
\left(T f_{n}\right)^{(k)}(0)=\left\{\begin{array}{cl}
1+2 / n & \text { if } \quad k=0 \\
k ! & \text { if } \quad k \geq 1
\end{array}\right.
$$

Hence $\left(T f_{n}\right)^{(k)}(0)$ does not converge to 0 for all $k$. Since $\Phi_{k}\left(0, \frac{e_{n}}{n}\right)=$ $\left(T f_{n}\right)^{(k)}(0)$, we also see that $\Phi_{k}(0, \cdot)$ is not bounded for any $k \in \mathbb{N} \cup\{0\}$.

\section{REFERENCES}

[1] J. Araujo, Realcompactness and spaces of vector-valued continuous functions, Fund. Math. 172 (2002), 27-40.

[2] J. Araujo, Realcompactness and Banach-Stone theorems, Bull. Belg. Math. Soc. Simon Stevin 11 (2004), 247-258.

[3] J. Araujo, Linear biseparating maps between spaces of vector-valued differentiable functions and automatic continuity, Adv. Math. 187 (2004), 488-520.

[4] J. Araujo, The noncompact Banach-Stone theorem, J. Operator Theory 55 (2006), 285-294.

[5] J. Araujo, E. Beckenstein and L. Narici, Biseparating maps and homoemorphic real-compactifications, J. Math. Anal. Appl. 192 (1995), 258-265.

[6] S. Banach, Théorie des Opérations Lineaires, Warszowa 1932. Reprinted, Chelsea Publishing Company, New York, 1963.

[7] E. Behrends, $M$-structure and the Banach-Stone Theorem, Springer- Verlag, Berlin, 1978.

[8] J.-X. Chen, Z.-L. Chen, And N.-C. Wong, A Banach-Stone Theorem for Riesz isomorphisms of Banach lattices, Proc. Amer. Math. Soc. 136 (2008), 3869-3874.

[9] J. Dugundu, Topology, Allyn and Bacon, Inc., Boston, 1966.

[10] Z. ERCAN AND S. ÖNAL, Banach-Stone Theorem for Banach lattice valued continuous functions, Proc. Amer. Math. Soc. 135 (2007), 2827-2829.

[11] Z. ERcan And S. Önal, The Banach-Stone Theorem revisited, Topology and its Applications 155 (2008), 1800-1803.

[12] M. I. Garrido And J. A. Jaramillo, Variations on the Banach-Stone theorem, Extracta Math. 17 (2002), 351-383.

[13] H.-W. Gau, J.-S. Jeang And N,-C. Wong, Biseparating linear maps between continuous vector-valued function spaces, J. Aust. Math. Soc. 74 (2003), 101-109.

[14] L. Gillman And M. Jerison, Rings of continuous functions. Graduate Texts in Mathematics, No. 43. Springer-Verlag, New York-Heidelberg, 1976.

[15] I. Gelfand and A. Kolmogorov, On rings of continuous functions on topological spaces, Dokl. Akad. Nauk. SSSR 22 (1939), $11-15$.

[16] J.-S. Jeang And N,-C. Wong, On the Banach-Stone problem, Studia Math. 155 (2003), 95-105. 
[17] I. Kaplansky, Lattices of continuous functions, Bull. Amer. Math. Soc. 53 (1947), $617-623$.

[18] S. LAng, Real analysis. Second Edition. Addison-Wesley Publishing Company, Advanced Book Program, Reading, MA, 1983.

[19] Ying-Fen Lin and Ngai-Ching Wong, The Structure of Compact Disjointness Preserving Operators on Continuous Functions, Math. Nach., to appear.

[20] H. H. Schaefer, Topological vector spaces. The Macmillan Co., New York, 1966.

[21] M. H. Stone, Applications of the theory of Boolean rings to general topology, Trans. Amer. Math. Soc. 41 (1937), $375-481$.

Department of Mathematics, National University of Singapore, 2 Science Drive 2, Singapore 117543.

E-mail address: matlhh@nus.edu.sg

Mathematics and Mathematics Education, National Institute of Education, Nanyang Technological University, 1 Nanyang Walk, Singapore 637616.

E-mail address: weekee.tang@nie.edu.sg 